NASA Technical Memorandum 104406

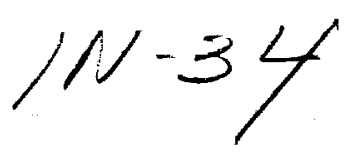

19772

843

\title{
Comparison of SMAC, PISO, and Iterative Time-Advancing Schemes for Unsteady Flows
}

Sang-Wook Kim

University of Texas at Arlington

Arlington, Texas

and

Thomas J. Benson

Lewis Research Center

Cleveland, Ohio

(NASA-TM-104400)

COMPAP I SON

ANO ITERATIVE TIME

UNSTEAUY FLOWS

(NASA)
$\mathrm{OF}$

SCHEMES

$43 \mathrm{P}$
N91-24550

Unclas

0019772

June 1991 


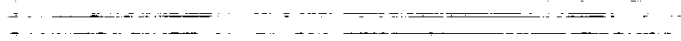
-..-- - -

$T+\cdots+\cdots$ - - . - .

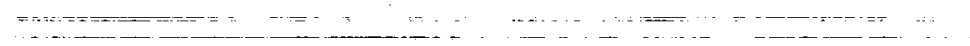

$\therefore-1=$

$-\cdots+\cdots=$

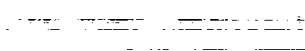

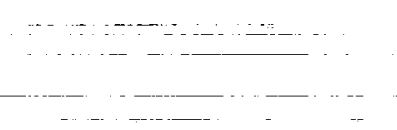

- - ....-
$-$

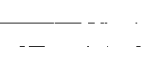




\title{
COMPARISON OF SMAC, PISO, AND ITERATIVE TIME-ADVANCING
}

SCHEMES FOR UNSTEADY FLOWS

\author{
Sang-Wook Kim* \\ University of Texas at Arlington \\ Department of Aerospace Engineering \\ Arlington, Texas 76010 \\ and \\ Thomas J. Benson \\ National Aeronautics and Space Administration \\ Lewis Research Center \\ Cleveland, Ohio 44135
}

\begin{abstract}
SUMMARY
Calculations of unsteady flows using a simplified marker and cell (SMAC), a pressure-implicit splitting of operators (PISO), and an iterative time-advancing scheme (ITA) are presented. A partial differential equation for incremental pressure is used in each time-advancing scheme. Example flows considered are a polar cavity flow starting from rest and self-sustained oscillatory flows over a circular and a square cylinder. For a large time-step size, the SMAC and ITA are more strongly convergent and yield more accurate results than PISO. The SMAC is the most efficient computationally. For a small time-step size, the three time-advancing schemes yield equally accurate strouhal numbers. The capability of each time-advancing scheme to accurately resolve unsteady flows is attributed to the use of a new pressure correction algorithm that can strongly enforce the conservation of mass. The numerical results show that the low frequency of the vortex shedding is caused by the growth time of each vortex shed into the wake region.
\end{abstract}

*Resident Research Associate at NASA Lewis Research Center. 
NOMENCLATURE

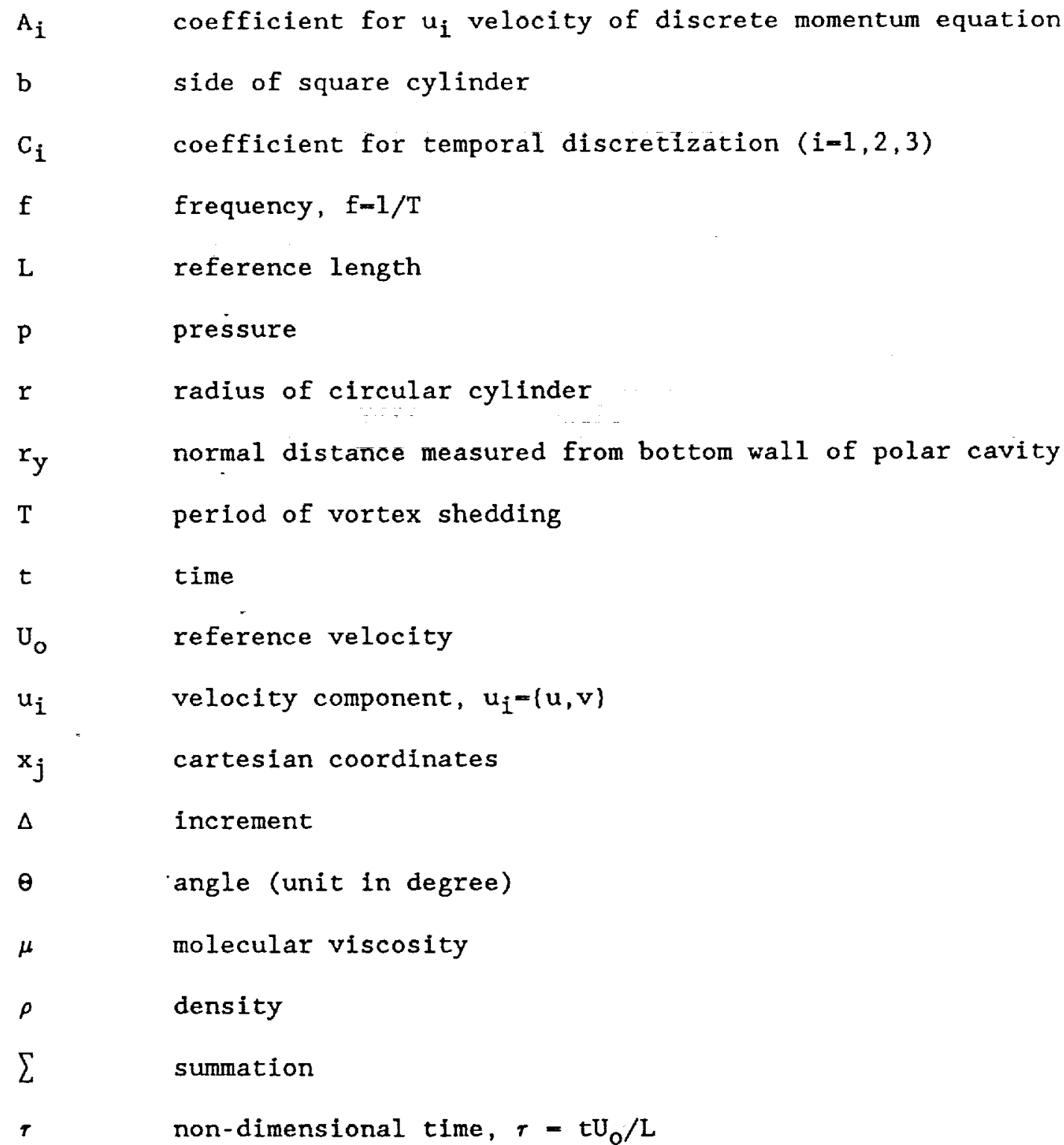

$\mathbf{n}$ time-level

subscript

nb 


\section{INTRODUCTION}

For incompressible flows, the conservation of mass acts as a constraint condition that the velocity field needs to satisfy; while in compressible flows, the conservation of mass is given as a partial differential equation for the temporal variation of density. Due to this distinct difference, recent developments of numerical methods to solve compressible flows are mostly concentrated on unsteady Navier-Stokes equations while those for incompressible flows are mostly concentrated on steady Navier-Stokes equations. Thus the numerical methods to solve unsteady incompressible flows have been reported only sporadically even though one of the earliest numerical methods to solve an unsteady, incompressible flow appeared as early as 1965 [1].

A careful examination of various unsteady incompressible flow solution techniques can provide a valuable guideline for a further extension of these numerical methods to solve more complex unsteady flow problems. The accuracy, convergence nature, and computational effort of SMAC [2], PISO [3,4] and ITA are examined by solving a polar cavity flow [5] starting from rest and self-sustained oscillatory flows over a circular cylinder [2] and a square cylinder [6].

The ITA is a direct extension of steady flow solution techniques to solve unsteady flows. In the method, a steady flow solution technique is used iteratively to obtain a converged solution for each time-level. Therefore, the ITA can be computationally heavy.

The marker and cell (MAC) scheme was originally developed to solve an unsteady free-surface flow. The method was simplified to solve an unsteady laminar flow over a circular cylinder by Braza et al. [2] and a few other flow cases cited in the reference. The name "marker and cell" 
has been retained in Ref. [2] and in the present study even though the numerical method itself has nothing to do with the "marker and cell" for the unsteady flows considered in these studies. Two slightly different schemes, varying in the complexity of the pressure equation, have been presented in Ref. [2]. The more complex case requires almost the same computational effort as that of PISO while the accuracy remains the same as that of the simpler case, and hence only the simpler case is considered herein. The simpler case is recast in a discrete form so that the context may be consistent with the other time-advancing schemes.

The PISO [3] was proposed to avoid the heavy computational effort of ITA. Calculations of a laminar flow through a suddenly expanding pipe and a compressible flow entering a suddenly expanding closed pipe can be found in [4]. In the present study, the PISO $[3,4]$ is modified to be memory efficient, which results in a slightly increased computational effort than the original PISO. For clarity, the modified PISO is described in detail in this paper.

In the pressure correction algorithm used in this paper, the incremental pressure is obtained by solving a partial differential equation for incremental pressure, which yields a strongly diagonally dominant system of equations for the incremental pressure. It has been shown in a number of numerical calculations of various two- and three-dimensional flows that the present method yields strongly and highly convergent results even when highly graded and skewed meshes are used to discretize the flow domain [7-10]. For example, the pressure correction algorithm yields a grid independent solution for a 3-D curved duct flow with a very small number of grid points and it can reduce the mass imbalance at least a few orders of magnitude smaller than those 
obtainable using various other pressure correction algorithms [9].

NUMERICAL METHODS

The incompressible laminar flow equations are given as;

$$
\begin{aligned}
& \frac{\partial u_{j}}{\partial x_{j}}=0 . \\
& \frac{\partial\left(\rho u_{i}\right)}{\partial t}+\frac{\partial}{\partial x_{j}}\left(\rho u_{i} u_{j}\right)-\frac{\partial}{\partial x_{j}}\left\{\mu\left(\frac{\partial u_{i}}{\partial x_{j}}+\frac{\partial u_{j}}{\partial x_{i}}\right)\right\}=-\frac{\partial p}{\partial x_{i}}
\end{aligned}
$$

where $\rho u_{i} u_{i}$ and $\rho u_{i} u_{j}(i \neq j)$ represent the nonlinearity in each component of the momentum equation and the nonlinear coupling between the $u_{i}$ - and $u_{j}$-velocity, respectively. Repeated indices imply summation over the index unless otherwise stated.

The unsteady flow solution techniques are implemented on a pressure-staggered mesh and the incremental pressure is obtained by solving a partial differential equation for incremental pressure. Only a few necessary details of the present pressure correction algorithm are summarized in "Iterative Time-Advancing Scheme" sub-section even though the same pressure correction algorithm is used for each of the unsteady flow solution techniques. Details on the pressure correction algorithm can be found in Refs. [7-9].

The transient term in the momentum equation is treated implicitly. The temporal variation of $\rho u_{i}$ can be discretized as

$$
\frac{\partial\left(\rho u_{i}\right)}{\partial t}=\rho C_{1} u_{i}^{n}-\rho C_{2} u_{i}^{n-1}+\rho C_{3} u_{i}^{n-2}
$$


where $\left(C_{1}, C_{2}, C_{3}\right)-(1 / \Delta t, 1 / \Delta t, 0)$ and $(3 / 2 \Delta t, 4 / 2 \Delta t, 1 / 2 \Delta t)$ for the first and the second order difference approximations, respectively. Since a few different time-advancing techniques are discussed below, the consistency of a few notations related to each time-advancing scheme is confined to each time-advancing scheme and each of such notations is explained only when it appears for the first time in each sub-section.

\section{Iterative Time-Advancing Scheme (ITA)}

The initial guesses for the new time-level velocity $\left(u_{1}^{*}\right)$ and pressure $\left(p^{*}\right)$ are set equal to those of the previous time-level. The discrete momentum equation based on the guessed flow variables can be written as

$$
\begin{aligned}
& \left(\rho C_{1}+A_{i}^{*}\right) u_{i}^{* *}=\sum_{n b}\left(A_{k}^{*} u_{k}^{* *}\right)+s_{i}^{*}-\frac{\partial p^{*}}{\partial x_{i}}+\rho C_{2} u_{1}^{n-1}-\rho C_{3} u_{i}^{n-2}, \\
& \text { no sum on } 1 \text {, }
\end{aligned}
$$

where $u_{1}^{* *}$ is a predicted velocity, $A_{i}^{*}$ is the coefficient of the $u_{i}^{* *}$-velocity, $s_{i}^{*}$ is a source term originating from a skewed mesh, and the coefficients $A_{i}^{*}$ and $S_{i}^{*}$ are evaluated using the initial guess. The predicted velocities are obtained by solving eq. (4). The predicted velocities are not necessarily divergence free, and hence the velocities are corrected to satisfy the conservation of mass. Let $u_{i}^{* * *}$ and $p^{\star *}$ are the corrected velocity and pressure that satisfy the conservation of mass. Then

$$
\begin{aligned}
& u_{i}^{* *}=u_{i}^{* *}+u^{\prime}{ }^{\prime} \\
& p^{* *}=p^{*}+p^{\prime}
\end{aligned}
$$


where $\mathrm{P}^{\prime}$ is the incremental pressure. The discrete momentum equations which satisfy the conservation of mass can be written as

$$
\begin{aligned}
& \left(\rho C_{1}+\stackrel{A_{i}^{*}}{*}\right) u_{i}^{* * *}-\sum_{n b}\left(A_{k}^{*} u_{k}^{* * *}\right)+s_{i}^{*}-\frac{\partial p^{* *}}{\partial x_{i}}+\rho C_{2} u_{i}^{n-1}-\rho C_{3} u_{i}^{n-2}, \\
& \text { no sum on } 1, \text { (7) }
\end{aligned}
$$

Subtracting eq. (4) from eq. (7) yields;

$$
u_{i}^{\prime}=-\frac{1}{\left(\rho C_{1}+A_{i}^{*}\right)} \frac{\partial p^{\prime}}{\partial x_{i}}, \quad \text { no sum on } i
$$

where the pressure gradient is left in continuous form deliberately. It is discussed later that the velocity-pressure decoupling that occurs when various pressure correction algorithms are used for a pressure-staggered mesh is caused by using a discretized pressure gradient in eq. (8) in deriving the discrete pressure correction equation. In deriving eq. (8), the summation over the neighboring grid points are disregarded. Retaining the residual originating from the neighboring grid points cause the numerical results to depend on the under-relaxation parameter [9]. Applying the conservation of mass to eq. (5) yields

$$
\frac{\partial u^{\prime} j}{\partial x_{j}}=-\frac{\partial u_{j}^{\star *}}{\partial x_{j}}
$$

Substituting eq. (8) into eq. (9) yields a partial differential equation for the incremental pressure given as;

$$
\frac{\partial}{\partial x_{j}}\left\{\frac{1}{\left(\rho C_{1}+A_{j}^{*}\right)} \frac{\partial p^{\prime}}{\partial x_{j}}\right\}-\frac{\partial u_{j}{ }^{*}}{\partial x_{j}}
$$


where the last term in eq. (10) represents the mass imbalance. The incremental pressure is obtained by solving eq. (10) and the velocities and pressure are corrected using eqs. (5) and (6), respectively. The above procedure needs to be iterated using $u_{i}^{* * *}$ and $p^{* *}$ as new initial guesses until the differences between the old and the new corrected velocities and pressures, respectively, become negligibly small; and the converged solutions represent the flow field of the current time-level.

A single iteration of the ITA can not account for the nonlinearity in the momentum equation. However, the flow equations are solved iteratively until the convergence criteria are met at each time-level, the nonlinearity in each component of the momentum equation and the nonlinear coupling of $u$-and $v$-velocity are fully accounted for in the method.

In each of the time-advancing schemes discussed in this paper, the incremental pressure is obtained by solving a partial differential equation for the incremental pressure. As all the central-differenced finite volume equations for self-adjoint second order elliptic partial differential equations are strongly diagonally dominant, the present discrete pressure correction equation is strongly diagonally dominant even for a highly skewed mesh. On the other hand, consider deriving a discrete pressure correction equation from eq. (8) using a discretized pressure gradient in eq. (8). Substituting eq. (8) into eq. (9) and integrating it over a pressure control volume yields a system of equations for incremental pressure which is not diagonally dominant. In such a case, the mass imbalance for a particular pressure grid point produces large pressure corrections for the adjacent pressure grid 
points, and the velocity-pressure decoupling occurs. In case a discrete pressure gradient is used in eq. (8) to derive a system of discrete pressure correction equations, only a fully-staggered mesh or the momentum interpolation schemes [11-12] can yield a diagonally dominant system of equations for the incremental pressure. However, the fully-staggered mesh is not an optimal grid layout for complex geometries; and the momentum interpolation scheme can not account for the grid skewness and it also yields a numerical result that depends on the under-relaxation parameter unless a specialized interpolation scheme is adopted $[9,12]$. However, the present pressure correction scheme does not yield a numerical result that depends on the under-relaxation parameter, since eq. (10) clearly states that the incremental pressure is driven only by the mass imbalance.

\section{Simplified Marker and Cell Scheme (SMAC)}

The SMAC and PISO are non-iterative time-advancing schemes and hence, an initial guess is not necessary for these schemes. The discrete momentum equations based on the flow variables of the previous time-level can be written as

$$
\begin{array}{r}
\left(\rho C_{1}+A_{i}^{n-1}\right) u_{i}^{*}=\sum_{n b}\left(A_{k}^{n-1} u_{k}^{*}\right)+s_{i}^{n-1}-\frac{\partial p^{n-1}}{\partial x_{i}}+\rho C_{2} u_{i}^{n-1}-\rho C_{3} u_{i}^{n-2}, \\
\text { no sum on } i
\end{array}
$$

where $u_{i}{ }^{*}$ is the predicted velocity, $A_{i}{ }^{n-1}$ is the coefficient of the $u_{i}{ }^{*}$-velocity, $s_{i}^{n-1}$ is a source term originating from a skewed mesh, and the coefficients $A_{i}^{n-1}$ and $s_{1}^{n-1}$ are evaluated using the flow variables of the previous time-level. The predicted velocities are obtained by 
solving eq. (11). The predicted velocity field may not satisfy the conservation of mass, thus the predicted velocities need to be corrected to satisfy the conservation of mass. Let $u_{i}{ }^{n}$ be the velocity that satisfy the conservation of mass. Then

$$
\stackrel{n}{u_{i}}-\stackrel{*}{u_{i}}=-\frac{\partial \phi}{\partial x_{i}}
$$

where $\phi$ is an auxiliary potential field. Taking divergence of eq. (12) yields

$$
\frac{\partial}{\partial x_{j}}\left\{\frac{\partial \phi}{\partial x_{j}}\right\}-\frac{\partial u_{j}^{*}}{\partial x_{j}}=0
$$

which can be solved in the same way as that for eq. (10). The divergence free velocity field for the current time-level is obtained using eq. (12). In the SMAC, the search for the flow field of the current time-level is concluded by obtaining a consistent pressure $\left(p^{\star}\right)$ that satisfies the momentum equation. The discrete momentum equation for the current time-level can be written as

$$
\left(\rho C_{1}+A_{i}^{n-1}\right) u_{i}^{n}=\sum_{n b}\left\{A_{k}^{n-1} u_{k}^{n}\right\}+s_{i}^{n-1}-\frac{\partial p^{n}}{\partial x_{i}}+\rho C_{2} u_{i}^{n-1}-\rho C_{3} u_{i}^{n-2}
$$

Subtracting eq. (11) from eq. (14) yields;

$$
\rho C_{1}\left(u_{i}^{n}-u_{i}^{\star}\right)=-\frac{\partial}{\partial x_{i}}\left(p^{n}-p^{n-1}\right)
$$

where $A_{i}{ }^{n-1}$ has been disregarded since $A_{i}{ }^{n-1}<<C_{1}$ for a very small 
time-step size. Substituting eq. (12) into (15) yields

$$
-\frac{\partial}{\partial x_{1}}\left(\rho C_{1} \phi\right)=-\frac{\partial}{\partial x_{1}}\left(p^{n}-p^{n-1}\right)
$$

or

$$
p^{n}=p^{n-1}+\rho C_{1} \phi
$$

where the spatial variation of $C_{1}$ has been neglected.

In eq. (14), the coefficients of the $u_{i}{ }^{n}$-velocity and the source term originating from a skewed mesh have not been updated. Thus SMAC can not account for the nonlinearity in each component of the momentum equation. It also can not account for the nonlinear coupling of $u$ - and $v$-velocity unless the discrete $u$ - and $v$-momentum equations are solved simultaneously as a single system of equations.

The velocity fleld obtained using the SMAC satisfy the conservation of mass strongly at each time-level. However, due to the use of an over-simplified pressure equation, eq. (16), the velocity and pressure fields may not satisfy the conservation of momentum rigorously. In fact, the over-simplified pressure equation can overshoot the pressure and it may yield a non-physical solution when the second order temporal discretization is used. On the other hand, the use of the first order temporal discretization always yields as accurate numerical results as those obtainable using the ITA or PISO incorporating the second order temporal discretization. This unusual behavior of the SMAC indicates that the second order temporal discretization is not quite compatible with the over-simplified momentum equation.

A more elaborate SMAC presented in Ref. [2] has also been tested in the course of the present study, however, any significant improvement in 
accuracy was not observed. The same observation has also been expressed in Ref. [2]. On the other hand, the computational effort of the alternative scheme becomes comparable to that of the PISO.

Pressure-Implicit Separation of Operators (PISO)

The discrete momentum equation based on the flow variables of the previous time-level can be written as

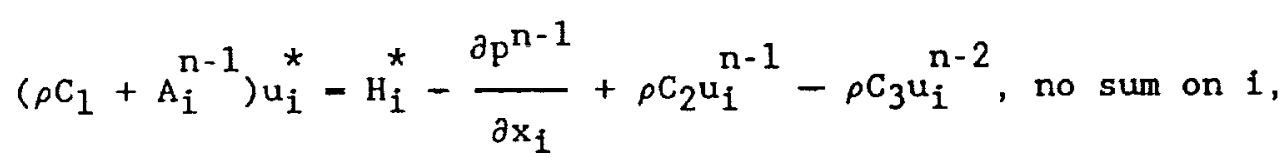

where

$$
H_{i}^{*}=\sum\left(A_{k}^{n-1} u_{k}^{*}\right)+s_{i}^{n-1}
$$

and the notations used in eq. (17) are the same as those for eq. (11). The predicted velocity is obtained by solving eq. (17). The predicted velocity field is not necessarily divergence free, and hence the velocity field needs to be corrected to satisfy the conservation of mass. Let $u_{i}^{* *}$ and $\mathrm{P}^{*}$ are the first corrected velocity and pressure that satisfy the conservation of mass, respectively. Then

$$
\begin{aligned}
& u_{i}^{\star *}=u_{i}^{*}+u_{i}^{\prime} \\
& p^{*}-p^{n-1}+p^{\prime}
\end{aligned}
$$

where $\mathbf{u}^{\prime}{ }_{i}$ and $\mathrm{p}^{\prime}$ are the first incremental velocity and pressure, respectively. The discrete momentum equations which satisfy the conservation of mass can be written as

$$
\left(\rho C_{1}+A_{i}^{n-1}\right) u_{i}^{* *}=H_{i}^{*}-\frac{\partial p^{*}}{\partial x_{i}}+\rho C_{2} u_{i}^{n-1}-\rho C_{3} u_{i}^{n-2} \text {, no sum on } i
$$


Subtracting eq. (17) from eq. (20) yields;

$$
u_{i}^{\prime}=-\frac{1}{\left(\rho C_{1}+A_{i}^{n-1}\right)} \frac{\partial p^{\prime}}{\partial x_{i}}, \text { no sum on } i
$$

where the summation over the neighboring grid points and the source term originating from a skewed mesh have been disregarded in deriving eq. (21). Applying the conservation of mass to eq. (18) yields

$$
\frac{\partial u^{\prime} j}{\partial x_{j}}=-\frac{\partial u_{j}^{*}}{\partial x_{j}}
$$

Substituting eq. (21) into eq. (22) yields a partial differential equation for the incremental pressure given as;

$$
\frac{\partial}{\partial x_{j}}\left\{\frac{1}{\left(\rho C_{1}+A_{j}^{n-1}\right)} \frac{\partial p^{\prime}}{\partial x_{j}}\right\}=\frac{\partial u_{j}^{*}}{\partial x_{j}}
$$

where the last term in eq. (23) represents the mass imbalance. The first incremental pressure is obtained by solving eq. (23), the first corrected pressure is obtained from eq. (19), and the first corrected velocity is obtained using eqs. (18) and (21). The first corrected velocity may satisfy the conservation of mass, but it does not satisfy the momentum equation according to Ref. [3,4]. Let the velocity and pressure that satisfy the momentum equation be $u_{1}{ }^{* *}$ and $p^{* *}$, where $u_{1}^{* * *}$ and $p^{* *}$ are the second corrected velocity and pressure, respectively. The discrete momentum equation based on the second corrected velocity and pressure can be written as 


$$
\left(\rho \mathrm{C}_{1}+\mathrm{A}_{i}^{\star *}\right) \mathrm{u}_{i}^{\star \star *}=\mathrm{H}_{i}^{* *}-\frac{\partial \mathrm{p}^{* *}}{\partial \mathrm{x}_{i}}+\rho \mathrm{C}_{2} \mathrm{u}_{\mathrm{i}}^{\mathrm{n}-1}-\rho \mathrm{C}_{3} \mathrm{u}_{\mathrm{i}}^{\mathrm{n}-2} \text {, no sum on } i \text {, }
$$

where

$$
\mathrm{H}_{\mathrm{i}}^{* *}=\sum_{\mathrm{nb}}\left[\mathrm{A}_{\mathrm{k}}^{* *} \mathrm{u}_{\mathrm{k}}^{* *}\right)+\mathrm{S}_{\mathrm{i}}^{\star *}
$$

$A_{i}{ }^{\star \star}$ and $S_{i}^{* \star}$ are evaluated using the first corrected velocity, and the momentum equation has been written in an explicit form to correct the velocity and pressure without solving a system of equations. Subtracting eq. (20) from eq. (24) yields;

$$
\left(\rho C_{1}+A_{i}^{* *}\right) u_{1}^{\prime \prime}=H_{i}^{* *}-H_{i}^{*}-\frac{\partial p^{\prime}}{\partial x_{i}} \text {, no sum on } i
$$

where $A_{1}{ }^{n-1}$ can be set equal to $A_{1}{ }^{\star \star}$ without incurring large error since $A_{j}^{* *} \ll \rho C_{1}$ and

$$
\begin{aligned}
& u_{i}^{\prime \prime}=u_{i}^{* \star}-u_{i}^{* *} \\
& p^{\prime \prime}=p^{\star *}-p^{*}
\end{aligned}
$$

are the second incremental velocity and pressure, respectively. Inserting eq. (25) into eq. (26) and taking divergence of eq. (26) yields;

$$
\frac{\partial}{\partial x_{j}}\left\{\frac{1}{\left(\rho C_{1}+A_{j}^{* *}\right)} \frac{\partial p^{\prime \prime}}{\partial x_{j}}\right\}=\frac{\partial}{\partial x_{j}}\left\{\frac{1}{\left(\rho C_{1}+A^{* *}\right\}}\left(H_{j}^{\star \star}-H_{j}^{*}\right)\right\},
$$

The second incremental pressure is obtained by solving eq. (28), the second corrected pressure is obtained from eq. (27), and the second corrected velocity is obtained from eqs. (25) and (26).

The present PISO algorithm is slightly different from that of Issa [3] in the load vector term of eq. (28). In the original PISO algorithm, 
the coefficients of the discrete momentum equation need to be stored to evaluate the load vector in eq. (29). In the present PISO algorithm, only the resultant given as $\mathrm{H}^{\star *}-\mathrm{H}^{*}$ needs to be stored to solve the second pressure correction equation. The present method is more memory efficient than the original PISO, while it requires more computational efforts. This modification has been motivated by the experience, obtained from numerical calculations of three-dimensional flows [9-10], that the required memory to solve complex three-dimensional flows may easily exceeds the current computer capacity. The modified PISO can better account for the nonlinearity in each component of the momentum equation since the updated residuals are used in solving the second pressure correction equation.

In the first corrector step of the PISO, the velocity and pressure are corrected to satisfy the conservation of mass. In the second corrector step, the velocity and pressure are corrected to satisfy the momentum equation. Therefore, the driving force of the second pressure corrector step is obtained from the momentum imbalance even though the pressure correction equation is still derived from the conservation of mass. Consequently, the velocity field at each time-level may not satisfy the conservation of mass very accurately and this forms the fundamental difference between the SMAC and PISO. As in the SMAC, the PISO algorithms can not accurately resolve the nonlinear coupling of the $u$-and v-momentum equations unless the discrete $u$ - and $v$-momentum equations are solved simultaneously as a single system of equations.

\section{NUMERICAL RESULTS}

Numerical results for the polar cavity flow [5] starting from rest 
and self-sustained unsteady flows over a circular cylinder [2] and a square cylinder [6] obtained using the ITA, SMAC and PISO are presented below. Calculations of the example flows using the ITA and PISO show that the second order implicit time-stepping scheme yields stable numerical results for the time-step size as large as ten times of that for the first order implicit time-stepping scheme. The numerical results presented in this section are obtained using the first order temporal discretization for the SMAC and the second order temporal discretization for the ITA and PISO.

The computational effort for each unsteady flow solution technique depends only slightly on each particular flow problem to be solved, the number of grid points and the number of time-steps, but it depends very strongly on the convergence criterla used. Since the system of equations are solved iteratively using a TDMA [13], one set of convergence criteria needs to be prescribed for the TDMA sweeps. The error norm used for the TDMA sweep is given as;

$$
|e|_{\ell}^{1}-\left|R_{k}, \ell / R_{0, \ell}\right|<\varepsilon_{\ell}^{1}
$$

where $\ell=\{u, v$, or $p\}$ denotes each velocity component and pressure, $R_{0}$ and $R_{k}$ are the sums of the absolute residuals of the discrete equation for every grid point evaluated at the Initial and at the $k$-th sweep of TDMA, and $\varepsilon_{\ell} l^{l}$ is the convergence criterion for the $\ell$-th flow variable. The SMAC and PISO are noniteartive time-advancing schemes, thus only the above set of convergence criteria needs to be prescribed. For the ITA, another set of convergence criteria is needed for the iterative solution of the flow equations. The error norm used for ITA is given as 


$$
|e|_{\ell}^{2}=\operatorname{MAX}\left(\operatorname{ABS}\left[\left(A_{k, \ell}^{n}-A_{k-1, \ell}^{n}\right) / A_{0, \ell}^{n} j \mid j-1, N\right)<\varepsilon_{\ell}^{2}\right.
$$

where $A^{n} o, l$ denotes the maximum $\ell$-th flow variable at the $n$-th time-level, $A_{k, l}^{n_{k}}$ is the $\ell$-th flow variable at the $k$-th iteration, and $N$ denotes the number of grid points. In the ITA, the flow variables at each time-level evolve iteratively, thus an accurate solution for each discrete system of equations at each iteration is not necessary. Hence a much smaller maximum number of TDMA-sweeps than those for the SMAC and PISO can be assigned to avoid excessive TDMA-sweeps that can be caused by eq. (29). The convergence criteria used are $\varepsilon_{\ell}^{1}=\left\{1 \times 10^{-7}, 1 \times 10^{-7}\right.$, $\left.1 \times 10^{-2}\right)$ and $\varepsilon \ell^{2}=\left(1 \times 10^{-2}, 1 \times 10^{-2}, 1 \times 10^{-2}\right)$. The numbers of maximum TDMA-sweeps for the SMAC and PISO are 11, 11, 100 for the discrete $u-$, v-, and p-equation, respectively; and those for ITA are 5, 5, 11 for the u-, v-, and p-equation, respectively. The maximum number of iterations for the ITA at each time-level is 11 . The computational efforts (CRAY/YMP cpu-time) per each grid point and per each time step for the above convergence criteria are shown in Table 1 . The use of slightly larger convergence criteria, smaller number of TDMA-sweeps and smaller number of iterations (for ITA) can decrease the computational effort significantly without losing the accuracy noticeably.

\section{Polar Cavity Flow Starting From Rest}

The lid-driven polar cavity flow is schematically shown in Fig. 1-(a). The Reynolds number based on the lid velocity and the depth of the cavity is 350 . The measured steady state velocity profiles can be found in Ref. [5]. The flow domain is discretized by $81 \times 81$ grid points. The evolution of $u_{\theta}$ at $\left(r_{y}, \theta\right)=(0.246,0.0)$ is shown in Fig. 1-(b). The 
normalized time for the polar cavity flow is based on the lid velocity and the depth of the polar cavity. For a large time-step size $(\Delta \tau=0.05)$, the SMAC yields a slightly unstable numerical result at $\tau \approx 1$ and the PISO does not yield a convergent solution. The calculated steady state velocity profiles are compared with the measured data in Fig. 1-(c). It is shown in the figure that the velocity profiles obtained using different time-advancing techniques collapse into a single line at each $\theta$-location and that the numerical results are in very good agreement with the measured data.

The mass imbalance for the polar cavity flow, obtained using the large time-step for each time-advancing scheme, is shown in Fig. 2. The mass imbalance produced by the ITA is one order of magnitude smaller than that produced by the SMAC. This result indicate that the ITA can most strongly enforce the conservation of mass and that the use of an under-relaxation do not obscure the numerical results. The ever-increasing mass imbalance produced by the PISO is mostly caused by the second corrector step for which the driving force is the momentum imbalance. For a small time-step size, the three methods yield equally accurate numerical results. However, the trend of the mass imbalance produced by the three methods remains the same as that shown in Fig. 2 .

\section{Vortex Shedding Behind a Circular Cylinder}

A laminar flow over a circular cylinder at Reynolds number 100 is considered below. The Reynolds number is based on the diameter of the cylinder and the free stream velocity. A survey of measured data and a set of numerical results obtained using the SMAC can be found in Ref. [2]. In Ref. [2], the flow equations transformed onto a cylindrical-polar 
coordinates were solved using a fully staggered mesh. In the present calculations, the inlet boundary is located at $8 \mathrm{r}$ upstream of the circular cylinder, the exit boundary is located at 110r downstream of the circular cylinder, and the side boundaries are located at $8 \mathrm{r}$ away from the circular cylinder. The flow domain is discretized by $146 \times 101 \mathrm{grid}$ points in $x-$, and $y$-coordinate directions, respectively. A uniform flow is prescribed at the inlet boundary and the vanishing gradient boundary condition is used at the exit boundary. A vanishing normal gradient of the tangential velocity and a vanishing transverse velocity are prescribed at the side boundaries. The flow field is perturbed by twisting the cylinder at the beginning so that the calculated velocity field can initiate the self-sustained oscillatory motion [2].

The streaklines calculated using the PISO are shown in Fig. 3. The streaklines show that the modified PISO can cleanly resolve the vortex shedding behind the circular cylinder.

The calculated Strouhal numbers are compared with the measured data as well as the numerical results by Braza et al. [2] and Eaton [14] in Table 2. The normalized time for flow over a circular cylinder is based on the free stream velocity and the radius of the cylinder. It can be seen in the table that the Strouhal number (for Reynolds number of 110) obtained using a penalty finite element method [14] is smaller than than the measured data. The Strouhal numbers obtained using the three time-advancing schemes, implemented on a pressure-staggered mesh and using the new pressure correction algorithm, are in very good agreement with the measured data.

The velocity vectors and the instantaneous streamilnes passing through the separation and/or reattachment locations for the flow over 
the circular cylinder is shown in Fig. 4. Instantaneous streamlines for unsteady flows are considered to be not meaningful by many researchers as yet. However, a close examination of the streamlines shown in Fig. 4 reveals that the mass flow rate across the streamlines are by far smaller than that along the streamlines. Thus the streamlines passing through the separation and the reattachment locations can be considered as an enclosure of the eddy attached to the cylinder. This interpretation of the instantaneous streamline is similar to that for steady flows. It can be seen in the figure that the attached vortex grows slowly until it becomes almost as large as the circular cylinder, and then the fully grown vortex is swept into the wake region by the nearby free stream. These figures clearly indicate that the low frequency (or the long period) of the vortex shedding is caused by the growth time of the attached vortex. It is also shown in the figure that each vortex shed into the wake region is accompanied by an alternating switching of the reattaching streamline with one of the two instantaneous streamlines passing through the separation locations. Undoubtedly, the instantaneous streamlines help to better understand the vortex shedding mechanism. It is also shown later in this section that the streamlines help to clearly identify the separation and reattachment locations.

The streamline contours and a carpet plot of stream function at $t=0.14 \mathrm{~T}$ are shown in Figs. $5-(a)$ and $5-(\mathrm{b})$, respectively. The saddle point shown in Fig. 5-(b) is caused by a closely spaced counter-ratating vortices, see Ref. [15] for more details.

The evolution of stagnation location in time for flow over a circular cylinder is shown in Fig. 6. It can be seen in Fig. 6-(b) that the numerical result obtained using the PISO exhibits a strong dependence 
on the time-step size. The amplitude and the phase difference obtained using the PISO with $\Delta t=0.05$ is in worst agreement with the other numerical results including the one obtained using the same PIso with $\Delta \tau=0.01$. As the time-step size is decreased, both numerical results obtained using the SMAC and PISO approach that obtained using ITA. These results indicate that the ITA yields the most accurate results due to its capability to resolve the nonlinearity in each component of the momentum equation and the nonlinear coupling of the $u$ - and v-velocity and the capability to enforce the conservation of mass most strongly. The strong dependence of the PISO on the time-step size is caused by the second corrector step which cause the velocity field less divergence free and by the linearized momentum equation that yields a solution regardless of the conservation of mass is rigorously satisfied or not.

The time-varying separation and reattachment locations are shown in Fig. 7-(a) and 7-(b), respectively. For the flow over the circular cylinder, the mesh and the time-step size are small enough to accurately resolve the vortices generated around the smooth cylinder. Thus, the numerical results obtained using the ITA and SMAC exhibit only a very small phase difference, while the PISO yields a numerical result that deviates most from the other numerical results. The reattachment location presented in Ref. [2] is, in fact, another separation point located at the symmetric lower part as shown in Fig. 4.

The lift and drag forces are shown in Figs. 8-(a) and 8-(b), respectively. As in Fig. 7, the numerical results obtained using the ITA and SMAC are in very good agreement with each other, while the PISO not only over-predict the amplitude but also yields a significant amount of phase difference. The present numerical methods yield slightly larger 
amplitudes for the lift and drag than those presented in Ref. [2].

\section{Vortex Shedding Behind a Square Cylinder}

A laminar flow over a square cylinder at Reynolds number 190 is considered below. The Reynolds number is based on a side of the square cylinder (b) and the free stream velocity. The measured strouhal number and a numerical result obtained using the ITA can be found in Ref. [6]. In Ref. [6], the flow domain was discretized using a fully staggered mesh and the convection terms were discretized using a formally third order accurate QUICKEST scheme. In the present calculations, the inlet boundary is located at $5.5 \mathrm{~b}$ upstream of the square cylinder, the exit boundary is located at $35 \mathrm{~b}$ downstream of the square cylinder, and the side boundaries are located at $5.5 b$ away from the square cylinder. The flow domain is discretized by $131 \times 101$ grid points in $x-$, and $y$-coordinate directions, respectively. The boundary conditions and the initial perturbations are the same as those used for the flow over a circular cylinder. For the flow over the square cylinder, the numerical methods yield the self-sustained oscillatory motion without the use of the initial perturbation. However, it takes a while for the fully oscillatory motion to be established.

The streaklines calculated using the SMAC are shown in Fig. 9. The streaklines show that the SMAC can cleanly resolve the vortex shedding behind the square cylinder.

The calculated Strouhal numbers are compared with the measured data as well as the numerical results obtained by Davis and Moore using the QUICKEST scheme [6] in Table 3. The normalized time for flow over the square cylinder is based on the free stream velocity and a side of the 
square cylinder. It can be seen in the table that the present numerical results are in very good agreement with the measured data, while the numerical results obtained by Davis and Moore deviate further as the mesh is refined. The calculated Strouhal number, which deviates farther from the measured data as the mesh is refined, is caused by the QUICKEST scheme [6].

The velocity vectors and the streamlines for the flow over the square cylinder is shown in Fig. 10. As in the flow over the circular cylinder, the attached vortex grows slowly until it becomes almost as large as the square cylinder, and then the fully grown vortex is swept into the wake region by the nearby free stream: Thus the vortex shedding mechanism and the cause for the low frequency vortex shedding are essentially the same as those for the flow over the circular cylinder. However, in the present case, the attached vortex is split into two parts by the sharp corner and hence, there almost always exists another pair of separation and reattachment locations than the flow over the circular cylinder.

The evolution of stagnation location in time for flow over a square cylinder is shown in Fig. 11. It can be seen in Fig. 11-(b) that the numerical results obtained using different time-advancing schemes yield small phase differences, but all the numerical results exhibit neatly organized oscillatory motion.

The time-varying lift coefficient for flow over the square cylinder is shown in Fig. 12. It can be seen in the figure that the amplitude obtained using the PISO with $\Delta r=0.05$ is in worst agreement with the other numerical results including the one obtained using the same PISO with $\Delta \tau=0.01$. As the time-step size is decreased, the amplitudes obtained 
using the SMAC and PISO approach that obtained using the ITA. Again, the undesirably strong dependence of the PISO on the time-step size is caused by the second corrector step which cause the velocity field to deviate further from a divergence free velocity field.

The drag for flow over the square cylinder is shown in Fig. 13. For the flow over the square cylinder, the time-step size is not small enough to accurately resolve the initially perturbed flow field. Thus, the numerical results obtained using the different time-advancing schemes exhibit large phase differences. However, all the numerical methods, except the PISO, yield almost the same frequencies and the amplitudes.

\section{CONCLUSIONS AND DISCUSSION}

Calculations of a polar cavity flow starting from rest and self-sustained oscillatory flows over a circular and a square cylinders using the ITA (Iterative Time-Advancing Scheme), SMAC (SImplified Marker and Ce11) and PISO (Pressure Implicit Splitting of Operators) are presented.

The numerical results show that the SMAC is the most efficient computationally and yields accurate results. Calculations of the lid-driven polar cavity show that the SMAC is even competetive with steady flow solvers to solve steady flows while the ITA, PISO and many other unsteady flow solvers are not $[9,15]$.

The ITA can account for the nonlinearity in each component of the momentum equation and the nonlinear coupling of the $u$ - and v-velocity through the iterative solution of the flow equations. Thus the ITA yields the most accurate numerical results for a large time-step size. The SMAC and PISO are non-iterative time-advancing schemes and hence these methods 
can only weakly account for the nonlinearity in the Navier-Stokes equations through the use of predictor and corrector steps. As the time-step size is decreased, the numerical results obtained using the SMAC and PISO approach those obtained using the ITA, which shows that the SMAC and PISO can accurately resolve the nonlinearity in the Navier-Stokes equations if a sufficiently small time-step is used.

The SMAC and PISO are quite different in their nature. In the SMAC, the predicted velocity field is corrected to satisfy the conservation of mass and the conservation of momentum is achieved by obtaining a consistent pressure. In the PISO, a second corrector step is introduced to correct the momentum imbalance. The numerical results show that the second corrected velocity field deviates farther from a divergence free velocity field and that the linearized momentum equation yields a solution regardless of the conservation of mass is rigorously satisfied or not. Thus the PISO exhibits an undesirable strong dependence on the time-step size and it is also weakly convergent. These numerical results indicate that accurately enforcing the conservation of mass is very important to enhance the convergence nature as well as to obtain accurate numerical results. The capability of each time-advancing scheme to accurately resolve the unsteady flows is largely attributed to the capability of the new pressure correction algorithm which can strongly enforce the conservation.

Numerical results for flows over a circular and a square cylinders show that a small vortex attached to the cylinder grows very slowly in time until it becomes as large as the cylinder and then the fully grown vortex is shed into the wake region. Each vortex shedding is accompanied by an alternating switching of the reattaching streamline with one of the 
two streamlines passing through the separation locations, and the alternating switching generates a small attached vortex. Each vortex shedding constitute a half cycle of the self-sustained oscillatory motion. The vortex leaving the cylinder is of the same size as that of the cylinder, and hence the low frequency is caused by the growth time of the attached vortex. 
REFERENCES

1. F. H. Harlow and J. E. Welch, "Numerical Calculation of Time-Dependent Viscous Incompressible Flow of Fluid with Free Surface," Physics of Fluids, vol. 8, pp. 2182-2189, 1965.

2. M. Braza, P. Chassing and H. Ha Minh, "Numerical Study and Physical Analysis of the Pressure and Vorticity Fields in the Near Wake of a Circular Cylinder," J.Fluid Mechanics, vol. 165, pp. 79-130, 1986.

3. R. I. Issa, "Solution of the Implicitly Discretized Fluid Flow Equations by Operator-Splitting," J. Computational Physics, vol. 62, pp. 40-65, 1986.

4. R. I. Issa, A. D. Gosman and A. P. Watkins, "The computation of Compressible and Incompressible Recirculating Flows by a Non-Iterative Implicit Scheme," J. Computational Physics, vol. 62, pp. $66-82,1986$.

5. L. Fuchs and N. Tillmark, "Numerical and Experimental Study of Driven Flow in a Polar Cavity," Int. J. Numer. Meth. Fluids, vol. 5, no. 4, pp. 311-329, 1985.

6. R. W. Davis and E. F. Moore, "A Numerical Study of Vortex Shedding from Rectangles," J.Fluid Mechanics, vol. 116, pp. 475-506, 1982.

7. S.-W. Kim, "Numerical Investigation of Separated Transonic Turbulent Flows with a Multiple-Time-Scale Turbulence Mode1," Numerical Heat Transfer, Part A, vol. 18, pp. 149-171, 1990.

8. S.-W. Kim, "Calculation of Reattaching Shear Layers in Divergent Channel with a Multiple-Time-Scale Turbulence Mode1," To appear in AIAA Journal, 1991.

9. S.-W. Kim, "Calculations of Separated 3-D Flows with a Pressure-Staggered Navier-Stokes Equations Solver, "NASA CR, In 
print, 1991.

10. S.-W. Kim and T. J. Benson, "Calculation of a Circular Jet in Crossflow with a Multiple-Time-Scale Turbulence Mode1," NASA TM, In print, 1991.

11. M Peric, R. Kessler and G. Scheurerer, "Comparison of Finite-Volume Numerical Methods with Staggered and Collocated Grids," Computers and Fluids, vol. 16, no. 4, pp. 389-403, 1988.

12. S. Majumdar, "Role of Underrelaxation in Momentum Interpolation for Calculation of Flow with Nonstaggered Grids," Numerlcal Heat Transfer, vo1. 13, pp. 125-132, 1988.

13. A. D. Gosman and F. J. K. Ideriah, "Teach-T," Dept. of Mech. Engrg., Imperial College, 1982.

14. B. E. Eaton, "Analysis of Vortex Shedding behind a Circular Cylinder by Computer-Aided Flow Visualization," J.Fluid Mechanics, vol. 180, pp. $117-145,1987$.

15. D. S. Jang, R. Jetli and S. Acharya, "Comparison of the PISO, SIMPLER, and SIMPLEC Algorithms for the Treatment of the Pressure-Velocity Coupling in Steady Flow Problems," Numerical Heat Transfer, vol. 10, pp. 209-228, 1986. 
TABLE I

Computational Efforts for Each Unsteady Flow Solution Method (CRAY/YMP cpu-time per each grid point and per each time step)

\begin{tabular}{|c|c|c|}
\hline ITA & SMAC & PISO \\
\hline $1.7 \times 10^{-4}$ & $2.5 \times 10^{-5}$ & $4.5 \times 10^{-5}$ \\
\hline
\end{tabular}

(unit in second)

TABLE II

Strouhal Numbers $\left(S_{t}=2 f r / U_{0}\right)$ for Flow over a Circular Cylinder

\begin{tabular}{|c|c|c|c|c|c|c|c|c|}
\hline & ITA & \multicolumn{2}{|c|}{ SMAC } & \multicolumn{2}{c|}{ PISO } & Ref. [2] & Ref. [14] & Exp't \\
\hline$\Delta \tau$ & 0.05 & 0.05 & 0.01 & 0.05 & 0.01 & 0.01 & - & - \\
\hline$S_{t}$ & 0.158 & 0.155 & 0.157 & 0.164 & 0.160 & 0.16 & 0.147 & 0.16 \\
\hline
\end{tabular}

TABLE III

Strouhal Numbers $\left(S_{t}-f b / U_{0}\right)$ for Flow over a Square Cylinder

\begin{tabular}{|l|l|l|l|l|l|l|l|l|}
\hline & ITA & \multicolumn{2}{|c|}{ SMAC } & \multicolumn{2}{c|}{ PISO } & \multicolumn{2}{c|}{ Ref. [6] } & Exp't \\
\hline$\Delta \tau$ & 0.05 & 0.05 & 0.01 & 0.05 & 0.01 & $0.05^{\mathrm{a}}$ & $0.05^{\mathrm{b}}$ & - \\
\hline $\mathrm{S}_{\mathrm{t}}$ & 0.133 & 0.126 & 0.13 & 0.142 & 0.135 & 0.159 & 0.165 & 0.146 \\
\hline
\end{tabular}

(a: $41 \times 40$ mesh, b: $51 \times 62$ mesh) 


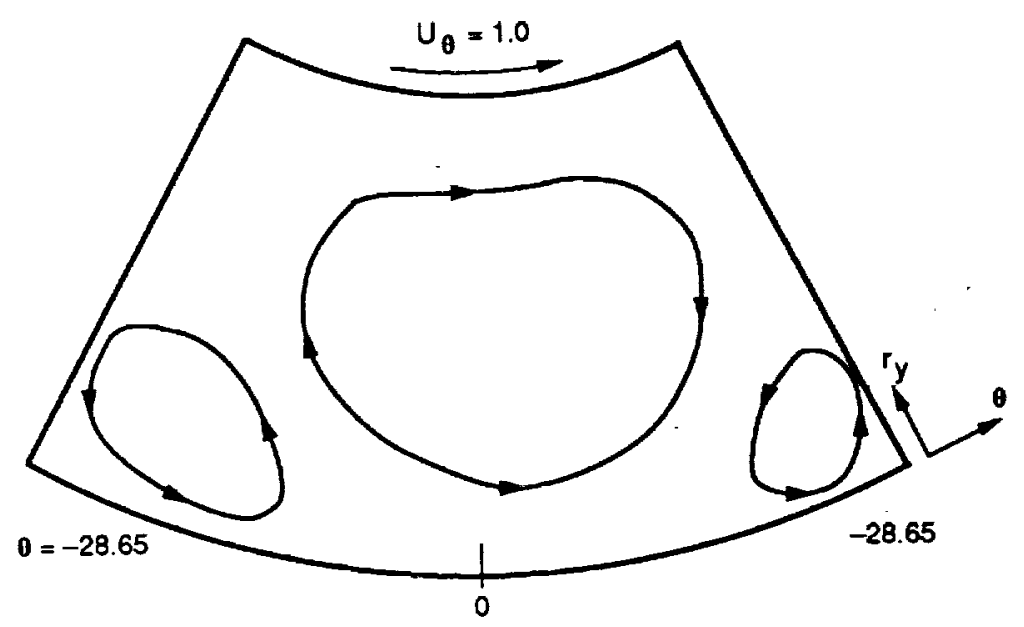

(a) Nomenclature.

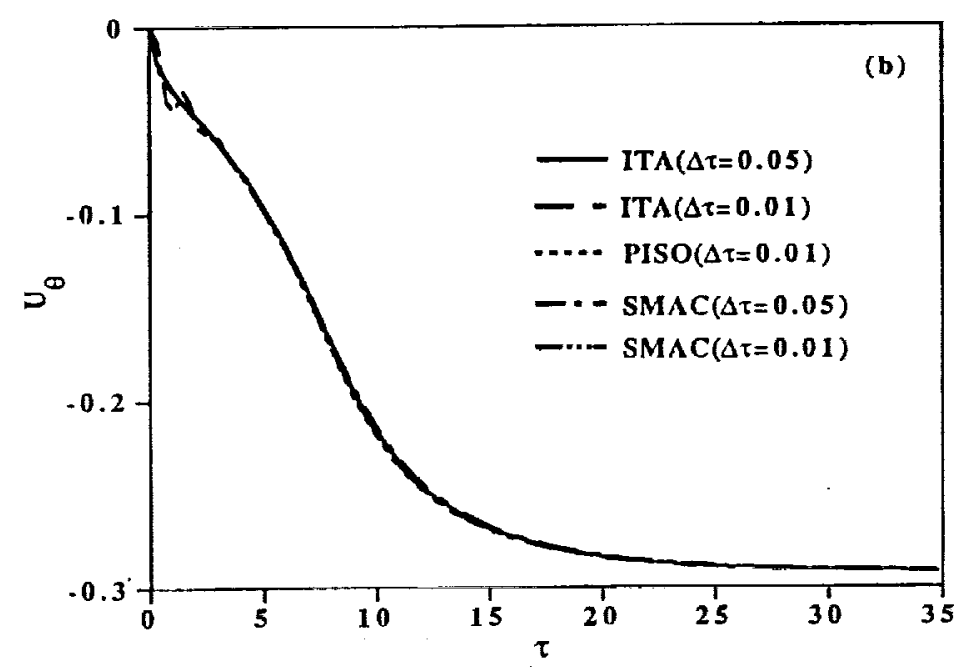

(b) Evolution of $u_{\phi}$ at $\left(r_{y}, \phi\right)=(0.246,0)$.

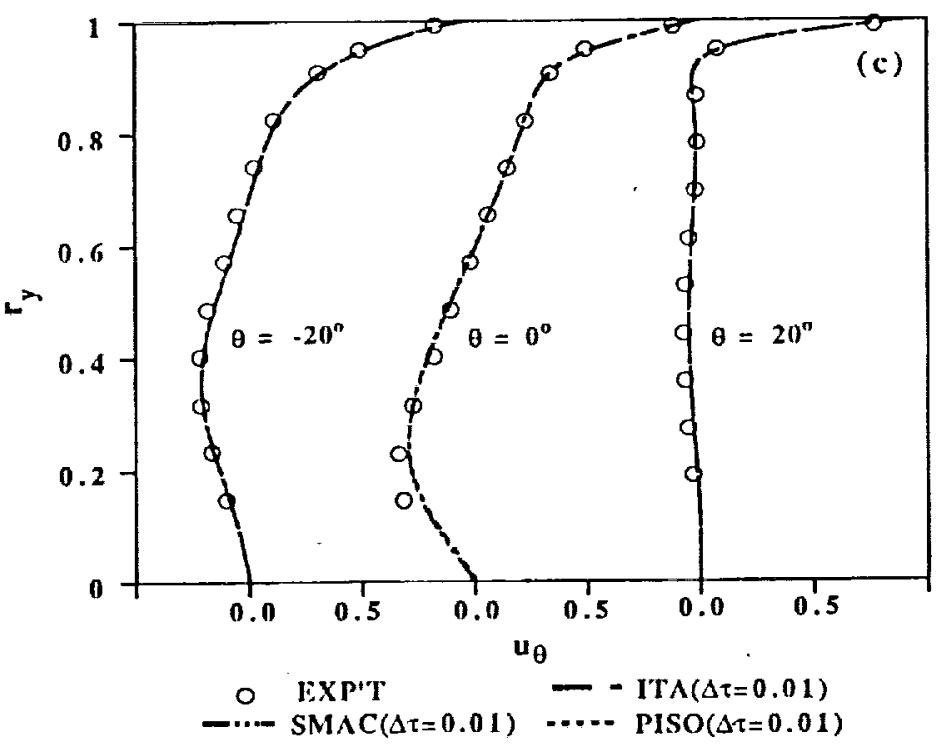

(c) $u_{\theta}$ velocily profiles.

Figure 1.- Polar cavity flow starting from rest. 


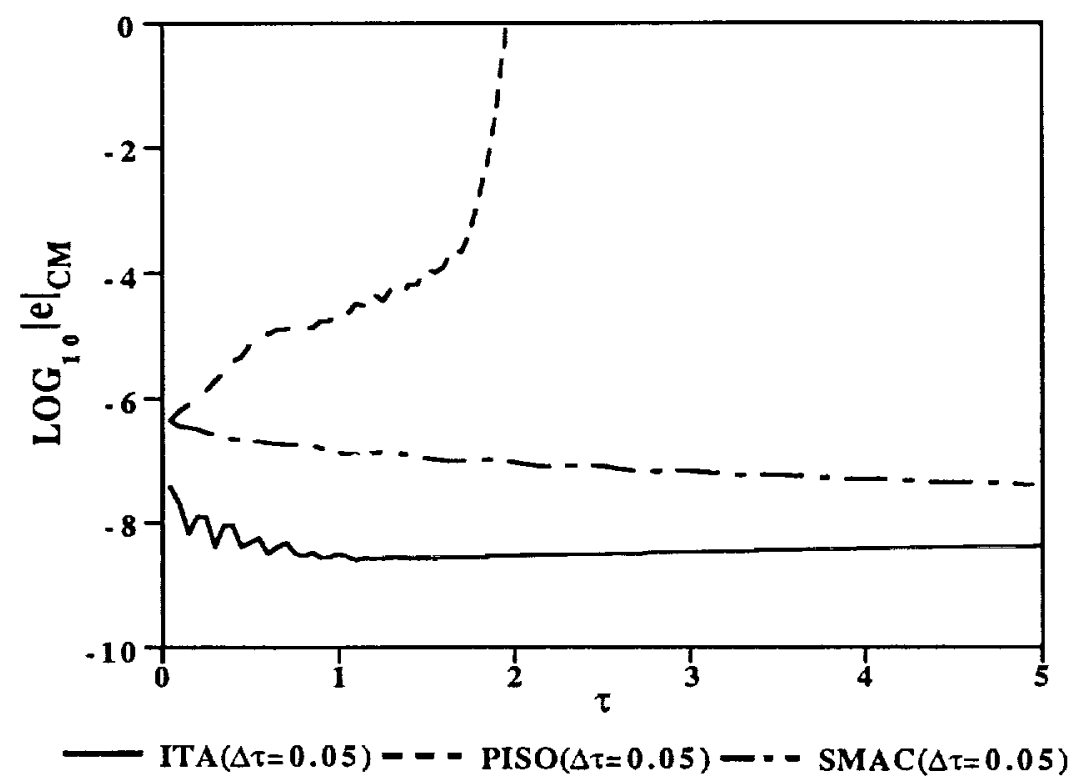

Figure 2.- Mass imbalance.

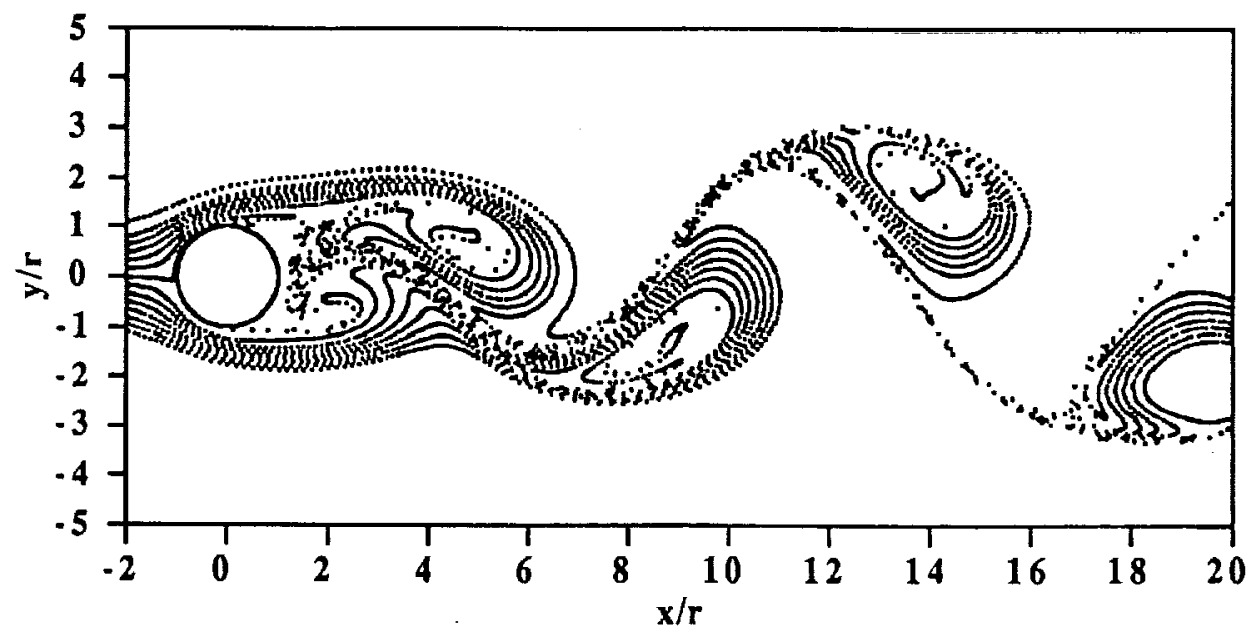

Figure 3.- Streaklines for flow over a circular cylinder. 

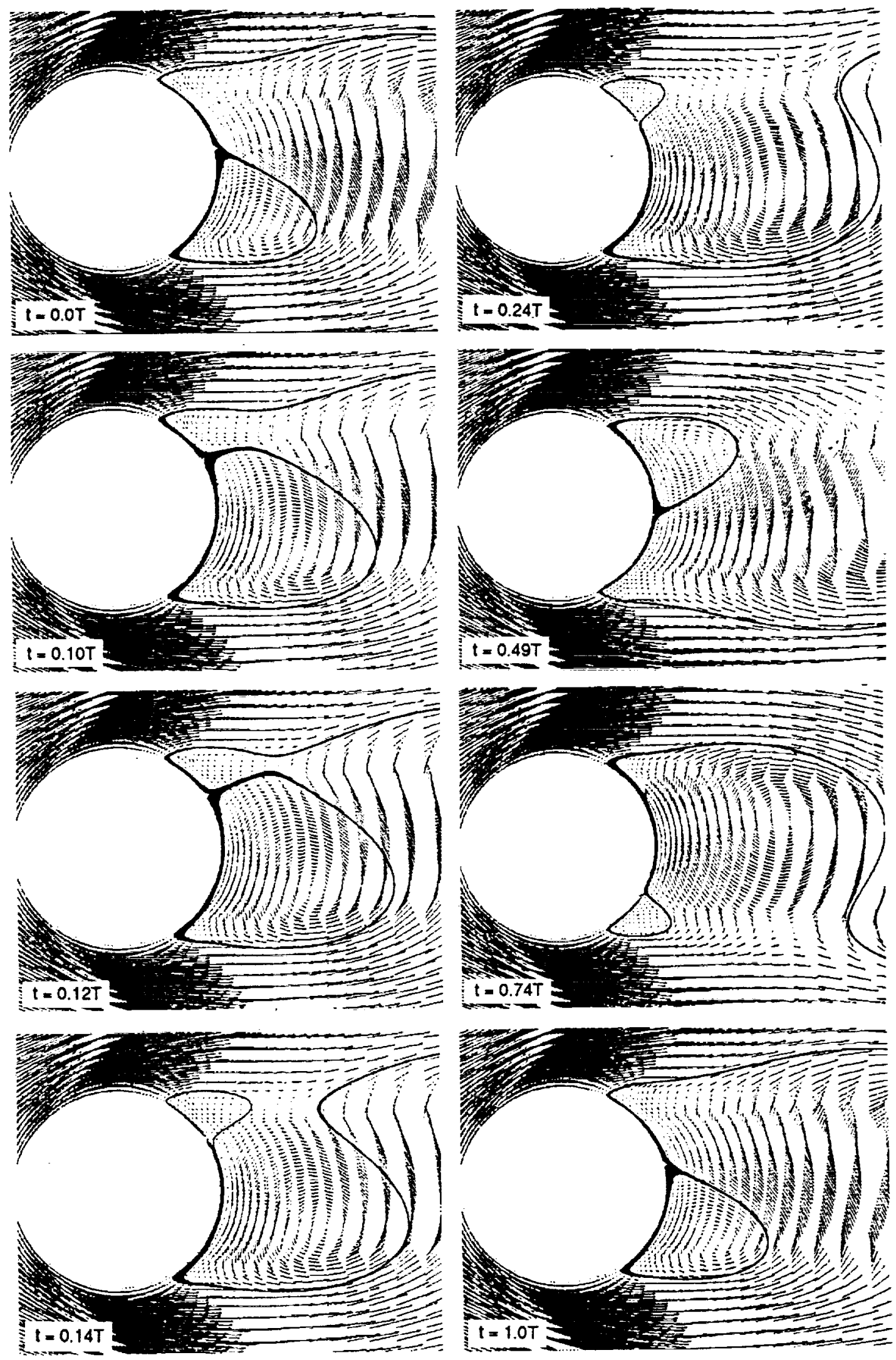

Figure 4.- Velocity vectors and instantaneous streamline contours passing through the separation and realtachment locations. 


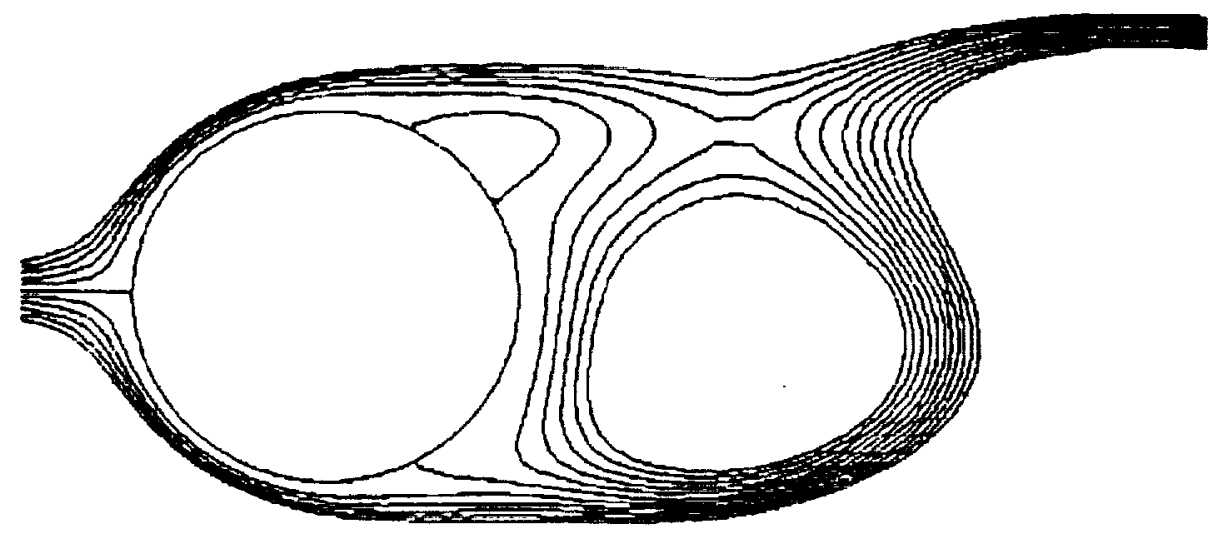

(a) Contour plot.

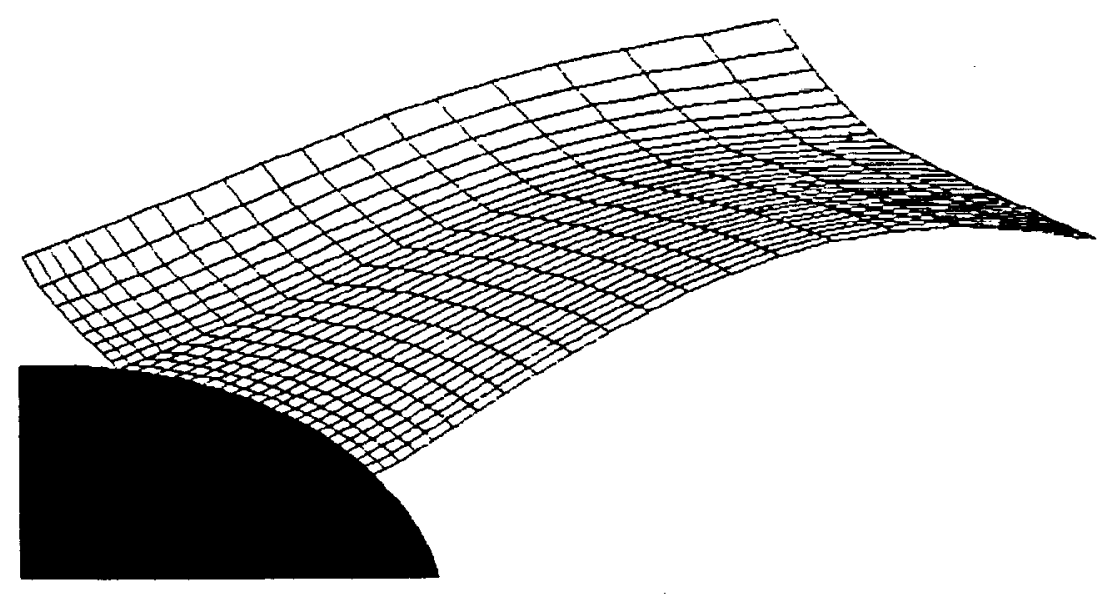

(b) Carpel plot.

Figure 5.- Streamlines at $\mathrm{t}=0.14 \mathrm{~T}$. 


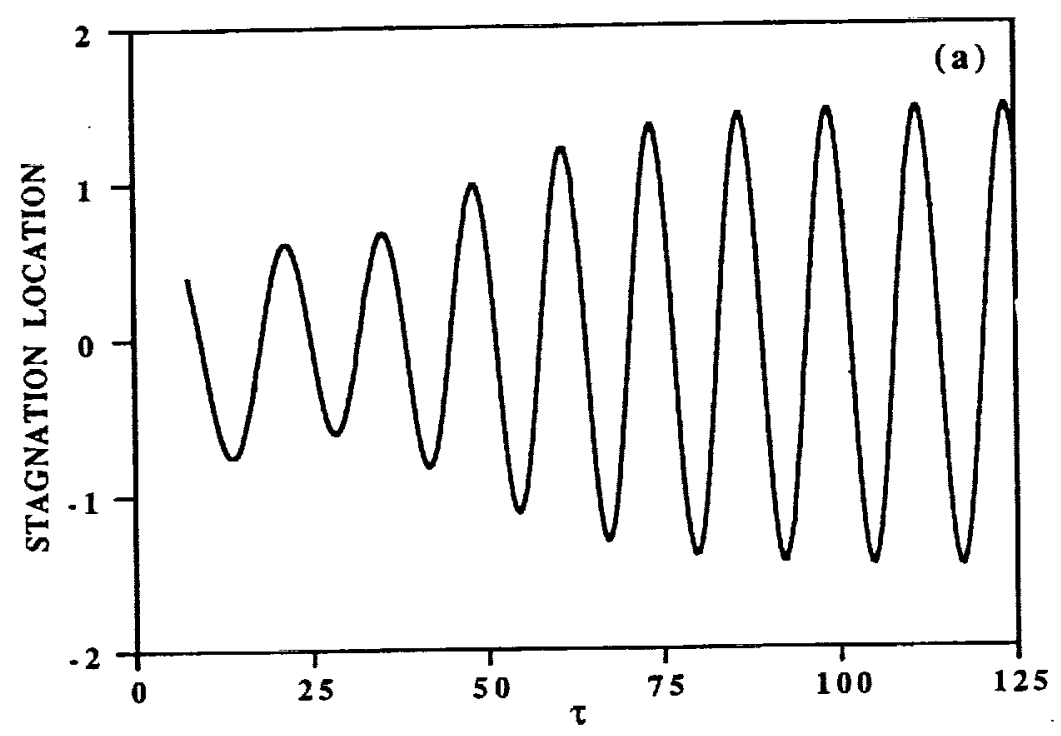

(a) PISO ( $\Delta r=0.01)$.

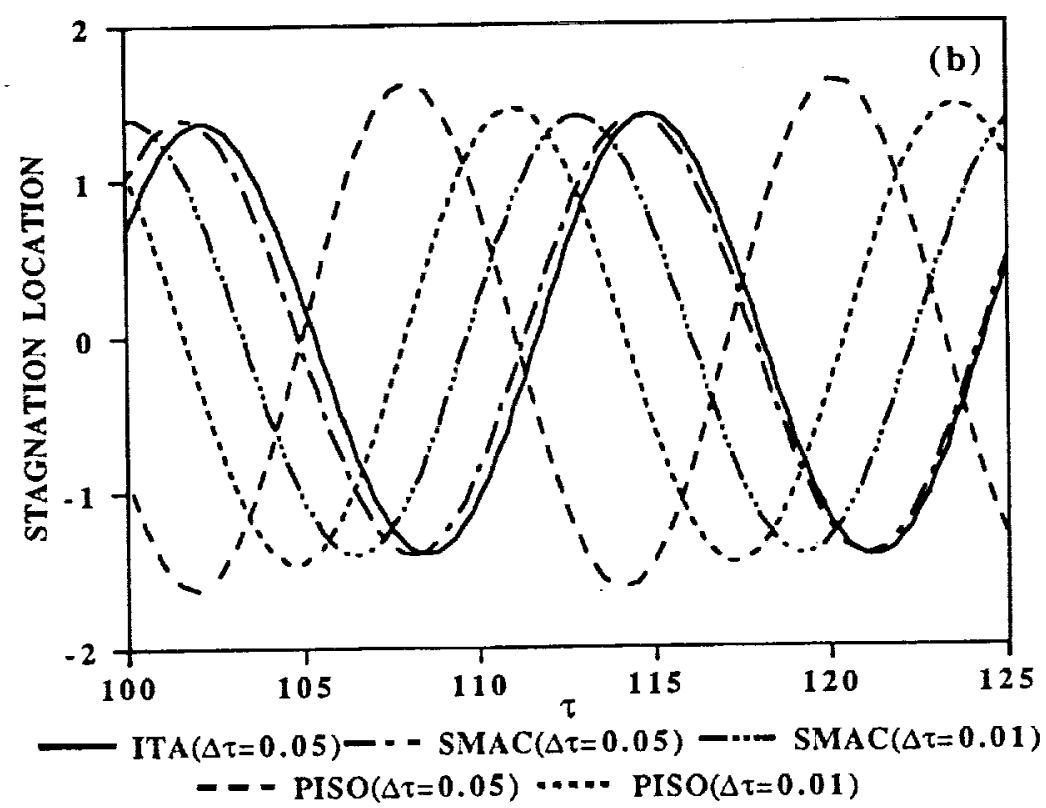

(b) Comparison of ITA, SMAC and PISO.

Figure 6.- Stagnation location for flow over a circular cylinder. 


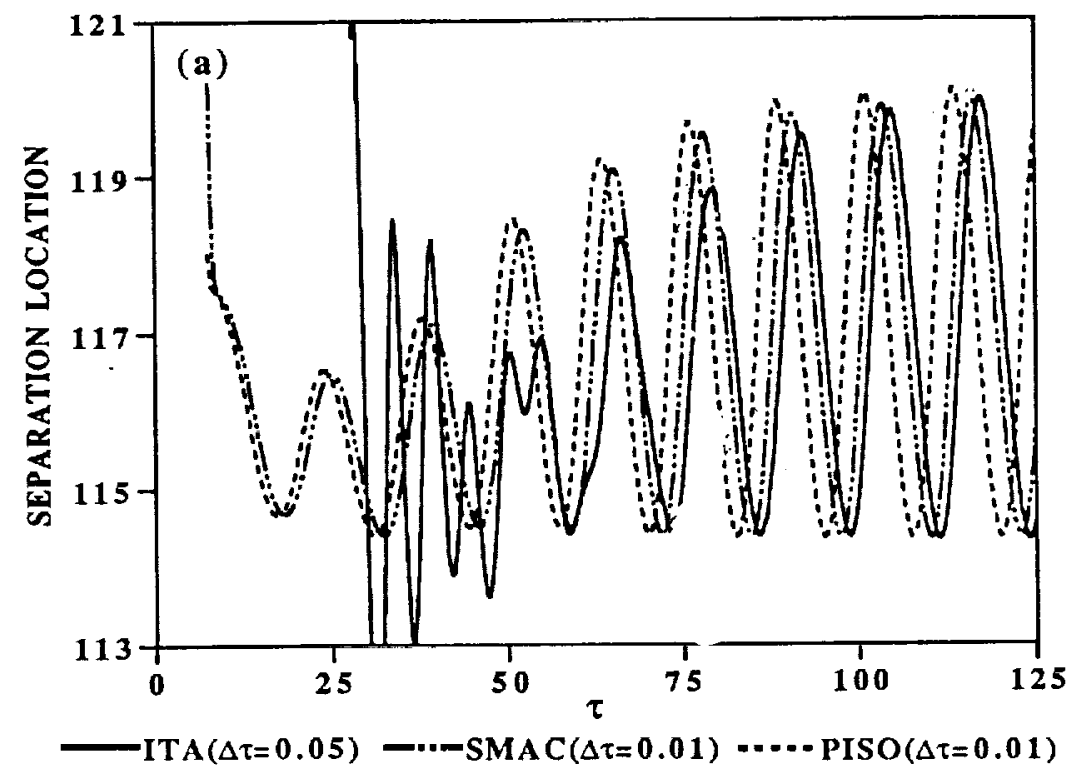

(a) Separation location.

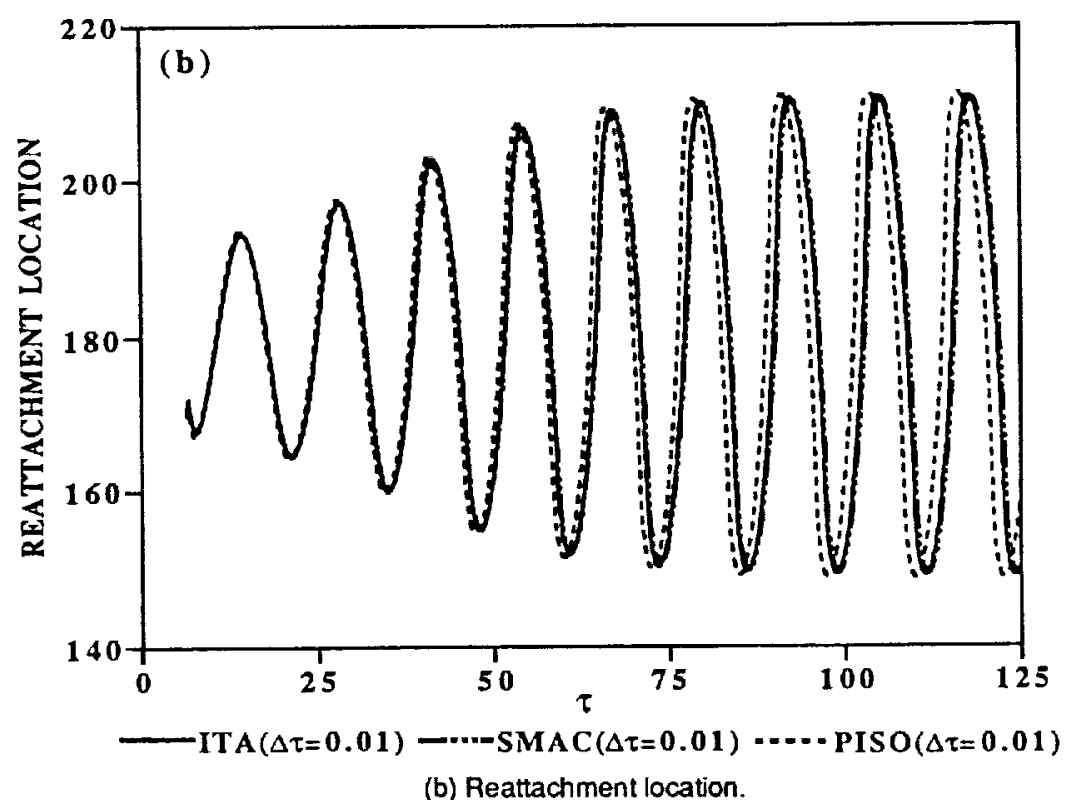

Figure 7.- Separation and reattachment locations for flow over a circular cylinder. 


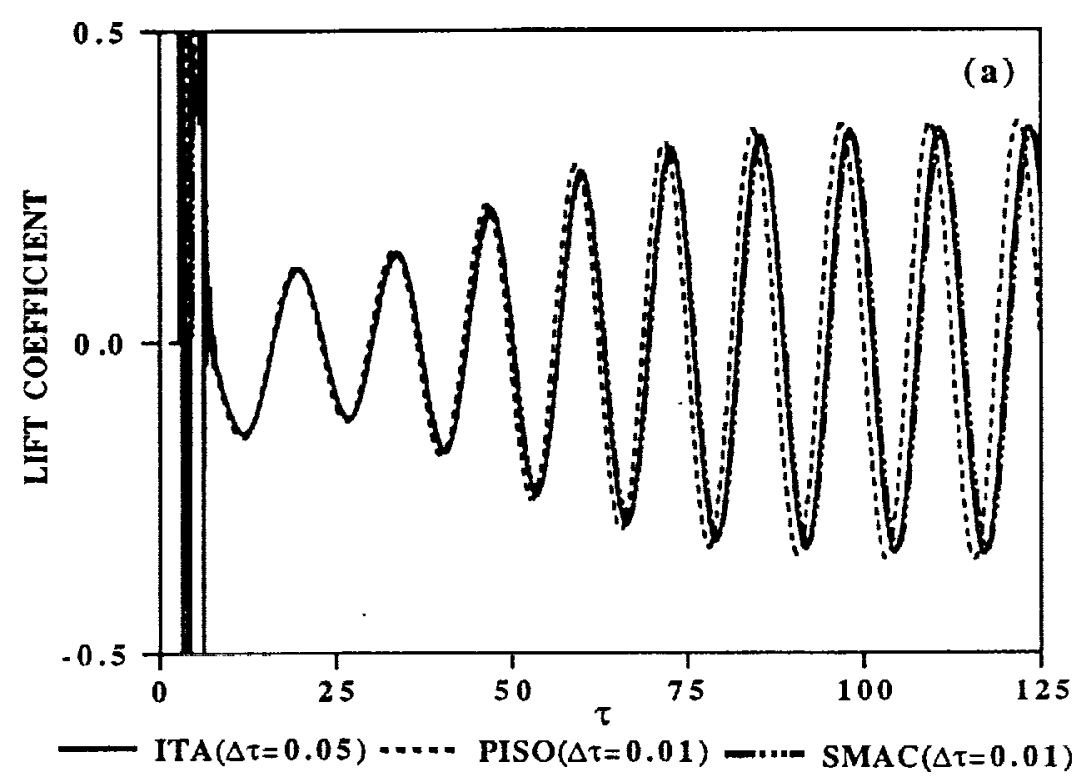

(a) Litt coefficient.

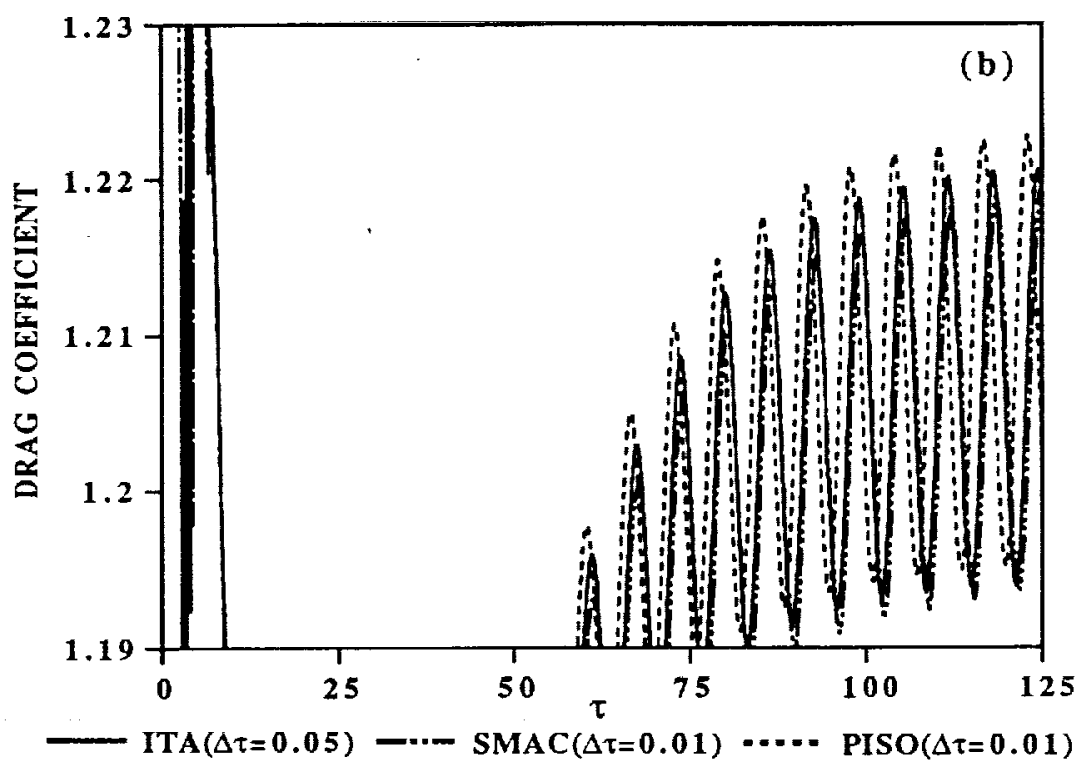

(b) Drag coefficient.

Figure 8.- Lift and drag for flow over a circular cylinder. 


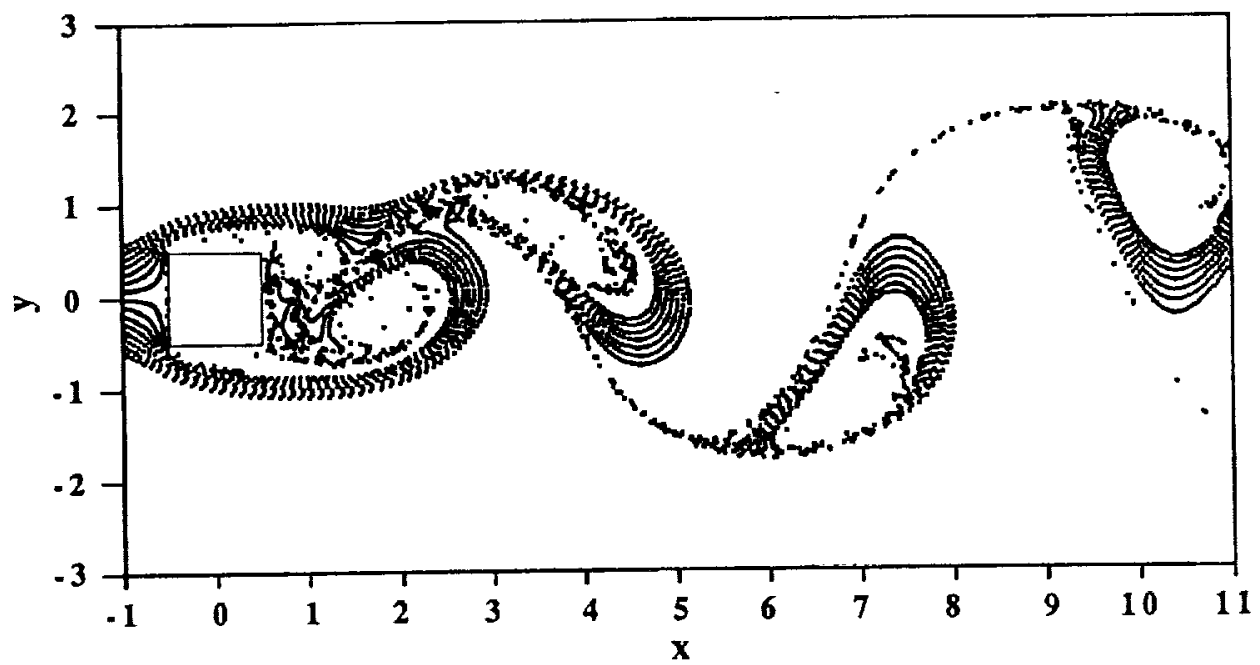

Figure 9.- Streaklines for flow over a square cylinder. 

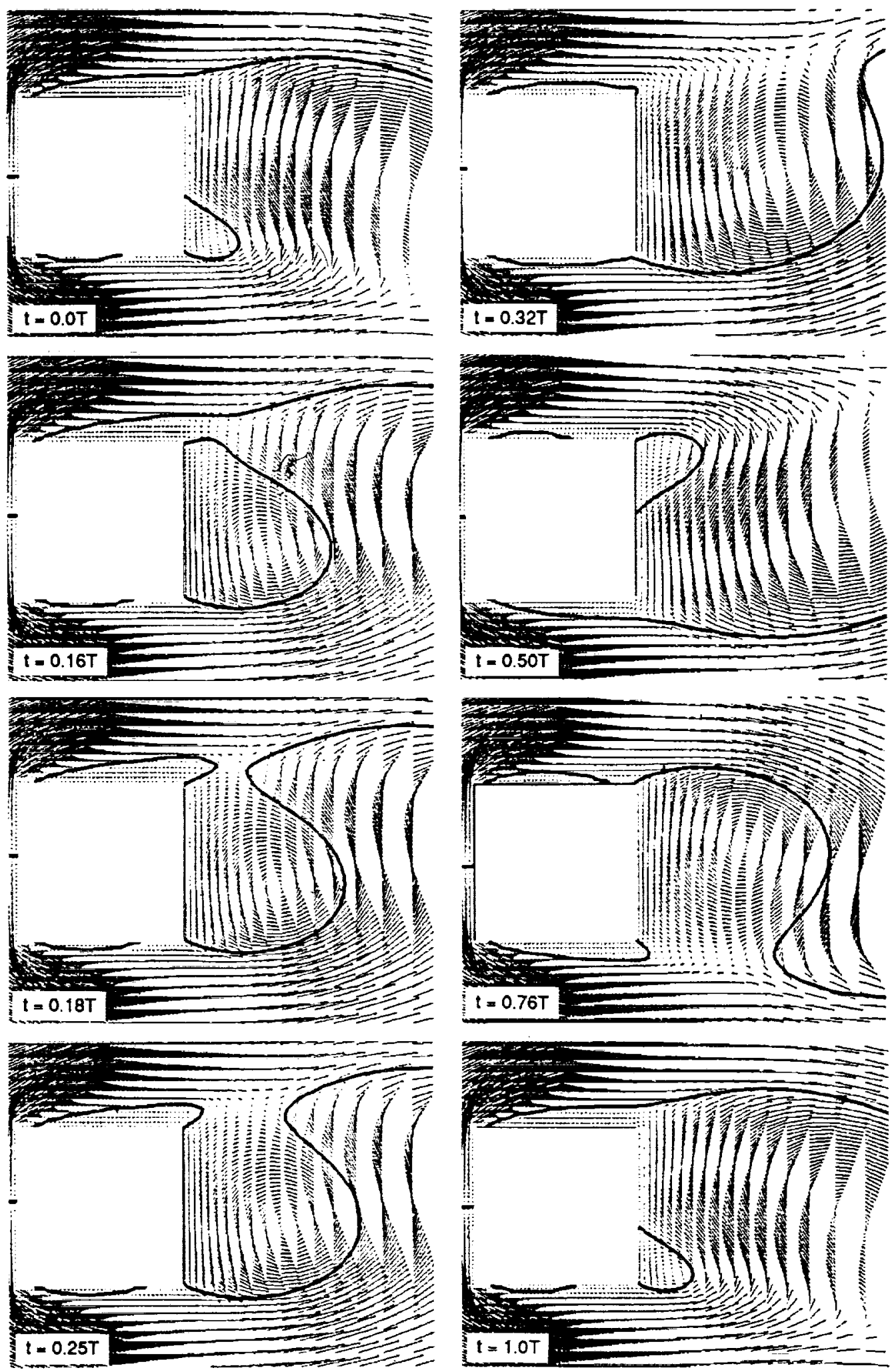

Figure 10.- Velocity vectors and instantaneous streamlines passing through the separation a reattachment locations. 


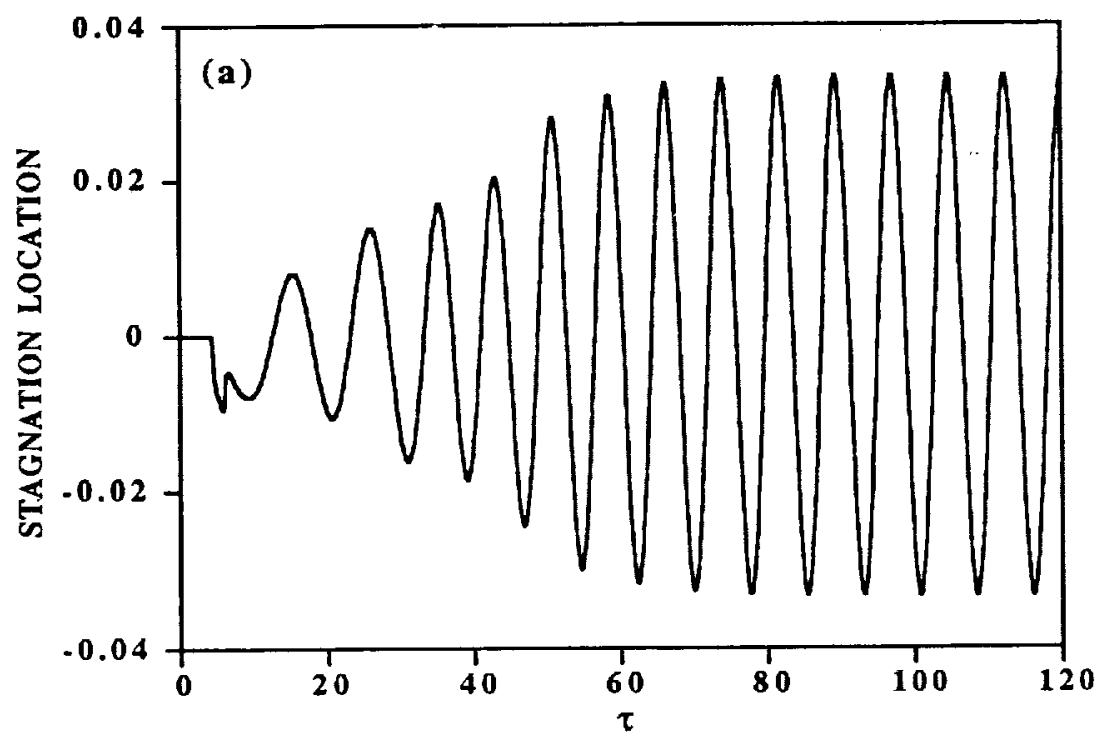

(a) SMAC $(\Delta r=0.01)$.

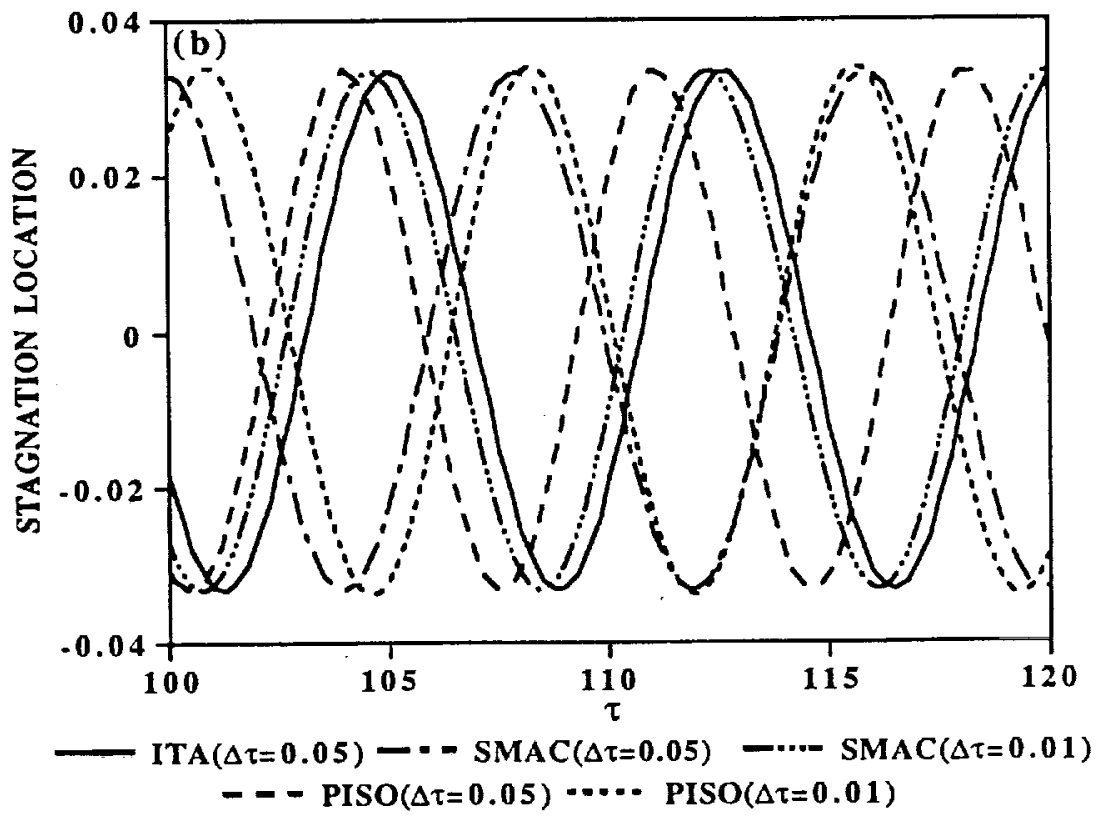

(b) Comparison of ITA, SMAC and PISO.

Figure 11.- Stagnation location for flow over a square cylinder. 


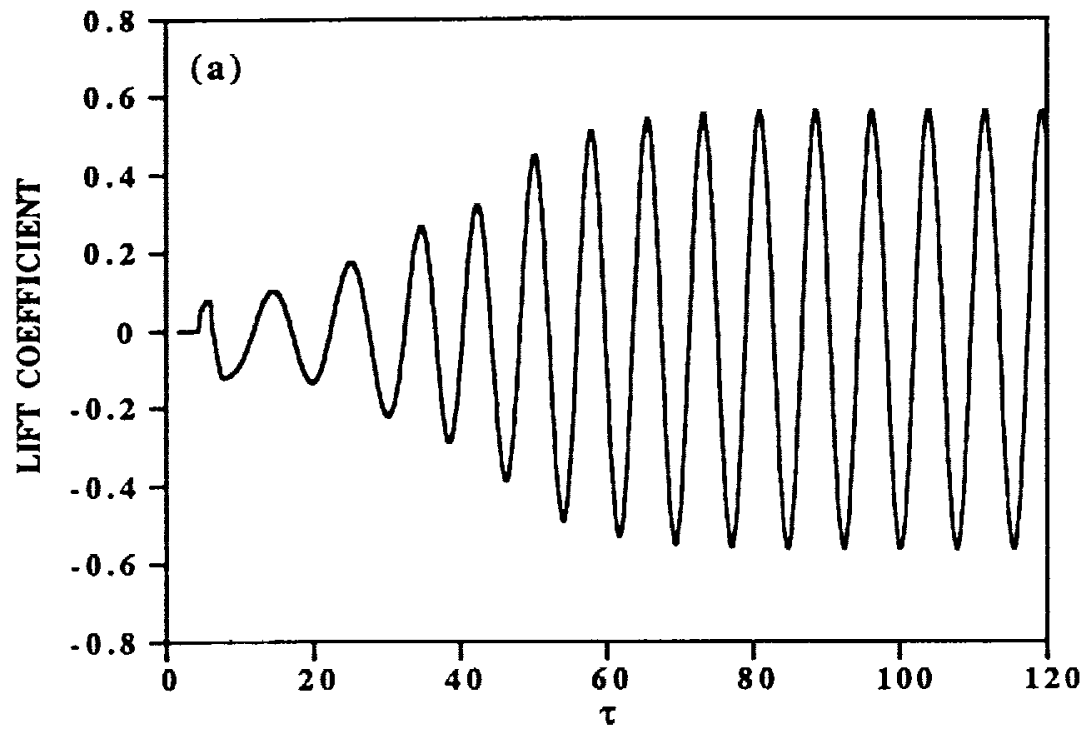

(a) SMAC $(\Delta r=0.01)$.

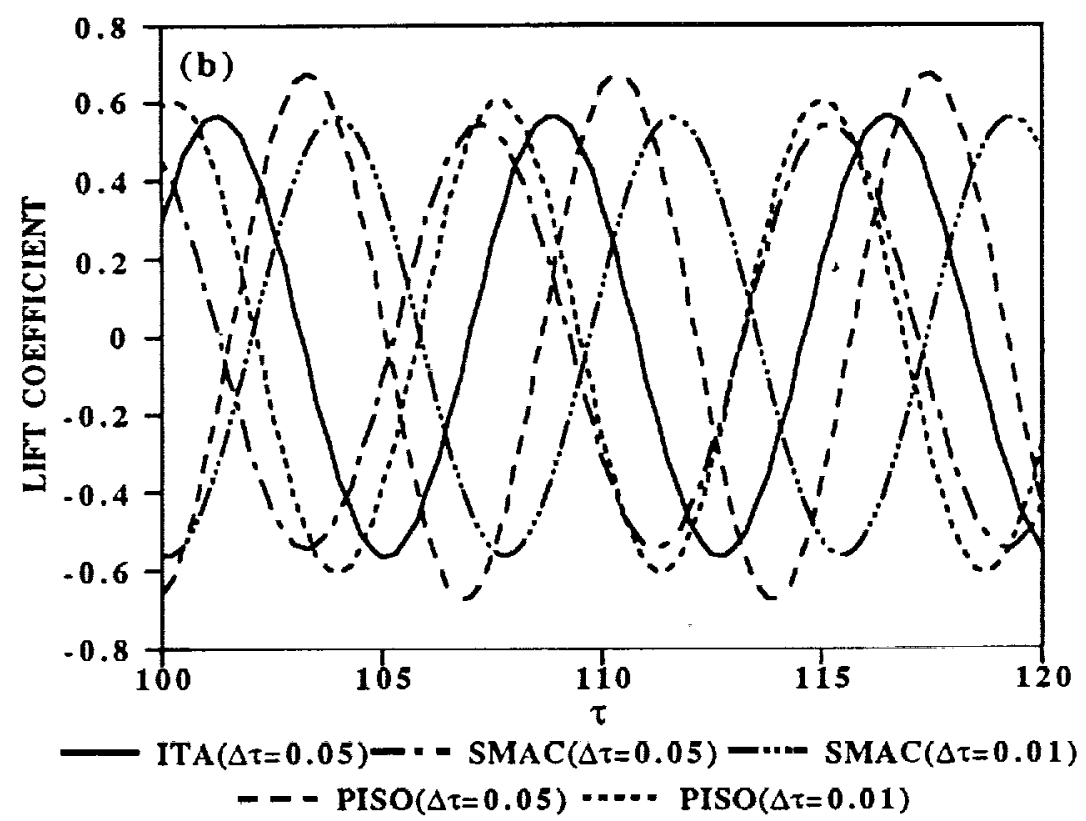

(b) Comparison of ITA, SMAC and PISO.

Figure 12.- Lift for flow over a square cylinder. 


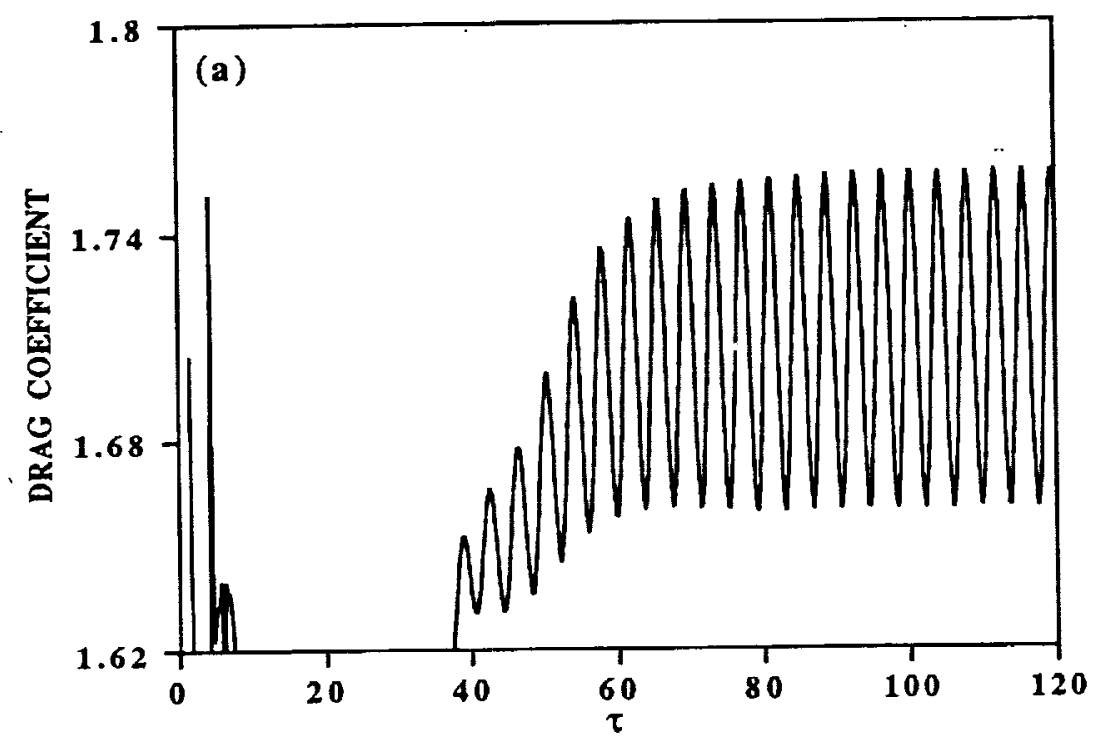

(a) SMAC $(\Delta r=0.01)$.

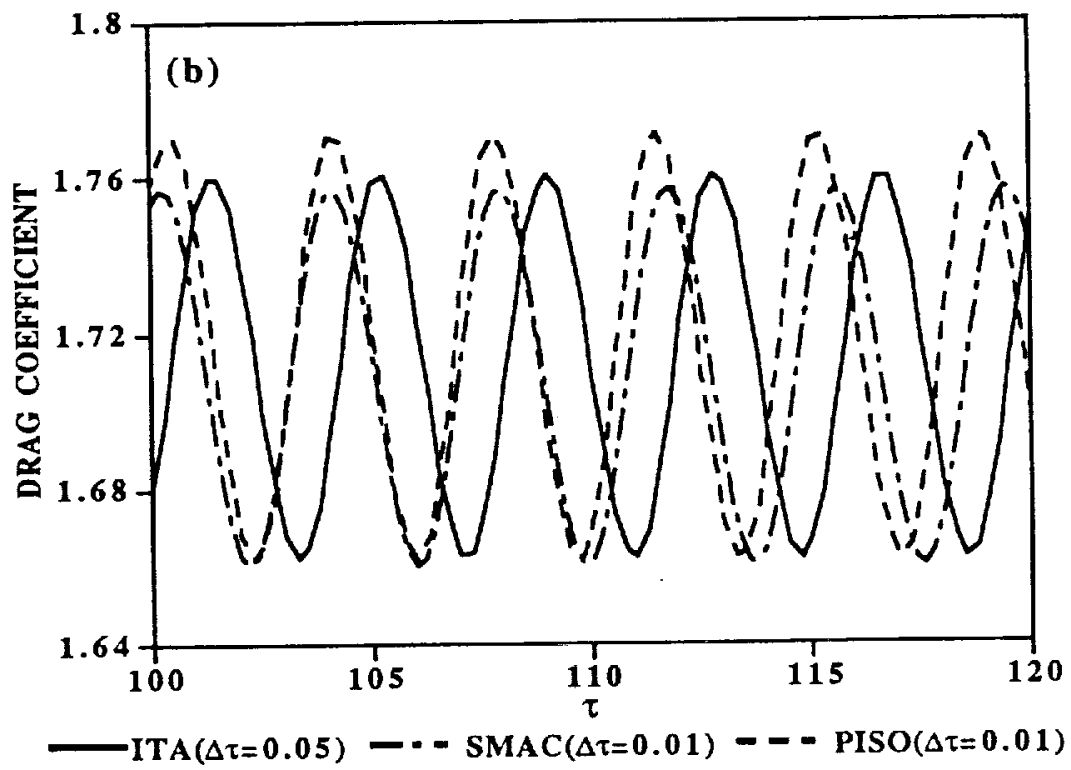

(b) Comparison of ITA, SMAC and PISO.

Figure 13.- Drag for flow over a square cylinder. 


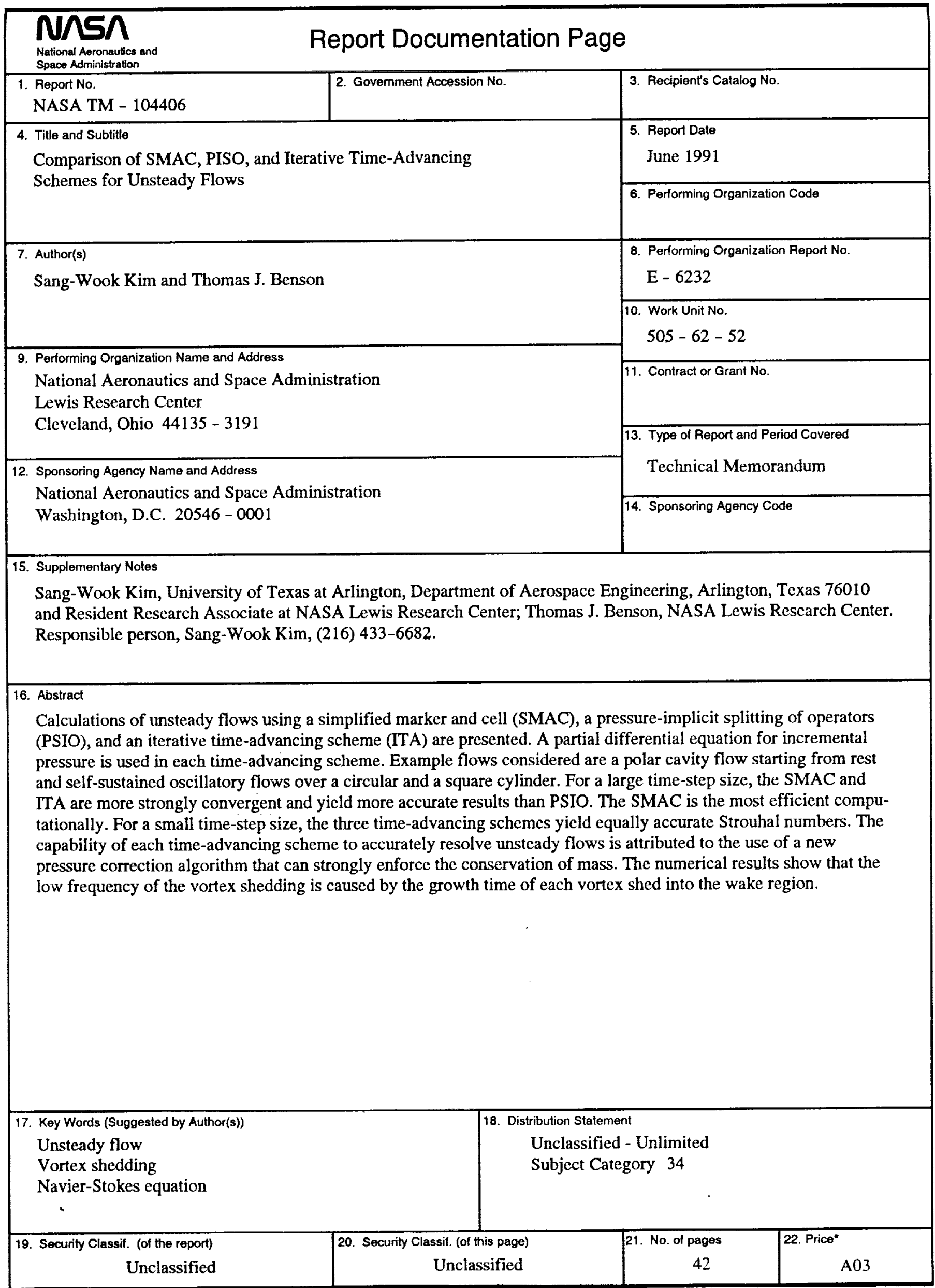

